\title{
On the heat diffusion for generic Riemannian and sub-Riemannian structures
}

\author{
Davide Barilari, Ugo Boscain, Grégoire Charlot, Robert W. Neel
}

July 2, 2018

\begin{abstract}
In this paper we provide the small-time heat kernel asymptotics at the cut locus in three relevant cases: generic low-dimensional Riemannian manifolds, generic 3D contact sub-Riemannian manifolds (close to the starting point) and generic 4D quasi-contact sub-Riemannian manifolds (close to a generic starting point). As a byproduct, we show that, for generic low-dimensional Riemannian manifolds, the only singularities of the exponential map, as a Lagragian map, that can arise along a minimizing geodesic are $A_{3}$ and $A_{5}$ (in the classification of Arnol'd's school). We show that in the non-generic case, a cornucopia of asymptotics can occur, even for Riemannian surfaces.
\end{abstract}

MSC classes: $53 \mathrm{C} 17 \cdot 57 \mathrm{R} 45 \cdot 58 \mathrm{~J} 35$

Keywords: generic sub-Riemannian geometry, heat kernel asymptotics, singularity theory.

\section{Introduction}

Let $M$ be a complete Riemannian or sub-Riemannian manifold. E Endow this structure with a smooth volume $\mu$ and consider the associated Laplace operator $\Delta$ defined as the divergence of the (horizontal) gradient. Under the assumption that the Hörmander condition is satisfied (which is obvious in the Riemannian case), and thanks to the completeness of $M$, the operator $\Delta$ is hypoelliptic and admits a smooth symmetric heat kernel $p_{t}\left(q_{1}, q_{2}\right)$ (see [21, 36]).

The problem of relating the small-time asymptotics of $p_{t}\left(q_{1}, q_{2}\right)$ with the properties of the (sub)Riemannian distance $d$ attracted attention starting from the 40s in the Riemannian case [28, 29], and then starting from the 80s for the sub-Riemannian one [13, 25, 26]. See [14, 35] for a historical viewpoint on the Riemannian heat equation, and [19] for a discussion about stochastic completeness of Riemannian manifolds.

In this paper we are interested in this problem "off-diagonal," namely when $q_{1} \neq q_{2}$. Let $\operatorname{Cut}\left(q_{1}\right)$ be the set of points where geodesics starting from $q_{1}$ lose their global optimality. Notice that, when there are no abnormal minimizers, $\operatorname{Cut}\left(q_{1}\right) \cup\left\{q_{1}\right\}$ (without $\left\{q_{1}\right\}$ in the Riemannian case) corresponds to the set of points where the function $d^{2}\left(q_{1}, \cdot\right)$ is not smooth. In 1988 Ben-Arous [13] proved that for $q_{2} \notin \operatorname{Cut}\left(q_{1}\right) \cup\left\{q_{1}\right\}$ and when there are no abnormal minimizers, one has the small-time heat kernel expansion (here $n$ is the dimension of the manifold)

$$
p_{t}\left(q_{1}, q_{2}\right)=\frac{C+O(t)}{t^{n / 2}} e^{-d^{2}\left(q_{1}, q_{2}\right) / 4 t}
$$

\footnotetext{
${ }^{1}$ In this paper, by sub-Riemannian manifold, we mean a constant rank sub-Riemannian manifold which is not Riemannian. By a (sub)-Riemannian manifold, we mean a constant-rank sub-Riemannian manifold, which is possibly Riemannian. However, several results of the paper hold for more general rank-varying structures in the sense of 11 , Appendix A], for instance, Grushin-like structures. This will be specified in the paper.
} 
for some $C>0$ depending on $q_{1}$ and $q_{2}$. This extended known results in the Riemannian context (see Molchanov [30], Azencott [10], Berger [14] and reference therein).

The behavior of $p_{t}\left(q_{1}, q_{2}\right)$ in the case $q_{2} \in \operatorname{Cut}\left(q_{1}\right)$ was a long-standing open problem. In Riemannian geometry it was investigated in [30], with further development in [32, 33, 11].

In the sub-Riemannian case, few properties of the cut locus were known before the mid-90s. Motivated by the need to better understand the structure of the cut locus, arising from the work of Ben Arous and Leandre, a precise description of the local structure of the cut locus for generic $3 \mathrm{D}$ contact structures and 4D generic quasi-contact structures was given by the control theory community, see [6, 17, 7, 16].

However the connection between the structure of the singularity and the heat kernel asymptotics remained unclear. Recently substantial progress in this direction has been made in [11, by adapting and further developing the techniques of Molchanov [30]. In particular, in [11] it was proved that the asymptotic is different from that of Equation (1) if and only if $q_{2}$ is cut-conjugate to $q_{1}$ (i.e. $q_{2} \in \operatorname{Cut}\left(q_{1}\right)$ and $q_{2}$ is conjugate to $q_{1}$ along at least one optimal geodesic). In this case we have the bounds, for small $t$ :

$$
\frac{C}{t^{n / 2+1 / 4}} e^{-d^{2}\left(q_{1}, q_{2}\right) / 4 t} \leq p_{t}\left(q_{1}, q_{2}\right) \leq \frac{C^{\prime}}{t^{n-(1 / 2)}} e^{-d^{2}\left(q_{1}, q_{2}\right) / 4 t},
$$

for some $C, C^{\prime}>0$ depending on $q_{1}$ and $q_{2}$. More precise estimates can be obtained if one has a precise description of "how much" $q_{2}$ is conjugate to $q_{1}$ (cf. Theorem 11).

The main purpose of this paper is to continue this analysis by giving the precise leading term for three cases of interests

(i) generic Riemannian manifolds of dimension less than or equal to 5 (starting from a fixed point).

(ii) generic 3D contact sub-Riemannian manifolds (for $q_{2}$ close enough to $q_{1}$ )

(iii) generic $4 \mathrm{D}$ quasi-contact sub-Riemannian manifolds (for $q_{2}$ close enough to $q_{1}$, with generic $\left.q_{1}\right)$

In the generic Riemannian case we stop at dimension 5 because up to dimension 5 the singularities of the exponential map are classified and they are structurally stable, see Section 1.1. As a byproduct of our analysis we get that the list of possible singularities arising generically on minimizing geodesics in the Riemannian case is rather restricted, see Theorem 5 below. Moreover we get a refinement of the main result [11, Theorem 27]. See Theorems 9 and 11.

The results given for cases (ii) and (iii) answer the question mentioned above about the heat kernel asymptotics at the sub-Riemannian cut locus which originally motivated many efforts by the control theory community in sub-Riemannian geometry.

In a complementary direction, we show that a variety of aymptotics can occur even in a twodimensional Riemannian manifold if one looks to non-generic cases.

For simplicity of exposition, to state our main results, we separate the Riemannian and subRiemannian cases.

\footnotetext{
${ }^{2}$ We recall that, in absence of abnormals, a geodesic cannot be conjugate before it reaches the cut locus.
} 


\subsection{The Riemannian case}

In this paper we always assume that the Riemannian metrics are complete. In addition, the following definition specifies what we mean by a generic Riemannian metric.

Definition 1. Let $M$ be a smooth manifold and $\mathcal{G}$ be the set of all complete Riemannian metrics on $M$ endowed with the $C^{\infty}$ Whitney topology. When we say that for a generic Riemannian metric on a manifold $M$ the property $(\mathrm{P})$ is satisfied we mean that the property $(\mathrm{P})$ is satisfied on an open and dense subset of the set $\mathcal{G}$.

In the following, $\mathcal{E}_{q}$ denotes the exponential map based at the point $q \in M$, thought as a map that, with an initial direction $v$ (i.e., $v \in T_{q} M,|v|=1$ ) and a time $t$, associates the end point $\mathcal{E}_{q}(t v)$ of the arc-length parameterised geodesic defined on $[0, t]$, starting at $q$ with direction $v$.

Conjugate points along geodesics correspond to singularities of the exponential map, which is a Lagrangian map. Thanks to the work of Arnol'd and his group (see [9] and references therein), we know the list of generic Lagrangian singularities up to dimension 5, which coincides with the list of stable Lagrangian singularities up to dimension 5.

Theorem 2 (Arnol'd's school: normal form of generic singularities of Lagrangian maps). Let $f$ be a generic Lagrangian map from $\mathbb{R}^{n}$ to $\mathbb{R}^{n}$ which is singular at a point $q$. Then, there exist changes of coordinates around $q$ and $f(q)$ such that in the new coordinates $q=0$ and:

- if $n=1$ then $f$ is the map $x \mapsto x^{2}$ (in this case the singularity is called $A_{2}$ );

- if $n=2$ then $f$ is the map $(x, y) \mapsto\left(x^{3}+x y, y\right)\left(A_{3}\right)$ or a suspension of the previous one;

- if $n=3$ then $f$ is the map $(x, y, z) \mapsto\left(x^{4}+x^{2} y+x z, y, z\right)\left(A_{4}\right)$, or $(x, y, z) \mapsto\left(x^{2}+y^{2}+x z, x y, z\right)\left(D_{4}^{+}\right)$, or $(x, y, z) \mapsto\left(x^{2}-y^{2}+x z, x y, z\right)\left(D_{4}^{-}\right)$, or a suspension of the previous ones;

- if $n=4$ then $f$ is the map

$(x, y, z, t) \mapsto\left(x^{5}+x^{3} y+x^{2} z+x t, y, z, t\right)\left(A_{5}\right)$, or $(x, y, z, t) \mapsto\left(x^{3}+y^{2}+x^{2} z+x t, x y, z, t\right)\left(D_{5}^{+}\right)$, or $(x, y, z, t) \mapsto\left(-x^{3}+y^{2}+x^{2} z+x t, x y, z, t\right)\left(D_{5}^{-}\right)$, or a suspension of the previous ones;

- if $n=5$ then $f$ is the map $(x, y, z, t, u) \mapsto\left(x^{6}+x^{4} y+x^{3} z+x^{2} t+x u, y, z, t, u\right)\left(A_{6}\right)$, or $(x, y, z, t, u) \mapsto\left(x^{4}+y^{2}+x^{3} z+x^{2} t+x u, x y, z, t, u\right)\left(D_{6}^{+}\right)$, or $(x, y, z, t, u) \mapsto\left(-x^{4}+y^{2}+x^{3} z+x^{2} t+x u, x y, z, t, u\right)\left(D_{6}^{-}\right)$, or $(x, y, z, t, u) \mapsto\left(x^{2}+x y z+t y+u x, y^{3}+x^{2} z+t x, z, t, u\right)\left(E_{6}^{+}\right)$, or $(x, y, z, t, u) \mapsto\left(x^{2}+x y z+t y+u x,-y^{3}+x^{2} z+t x, z, t, u\right)\left(E_{6}^{-}\right)$, or a suspension of the previous ones. 
Here by a suspension of a map $f: \mathbb{R}^{n} \rightarrow \mathbb{R}^{n}$ we mean a map of the form $\tilde{f}: \mathbb{R}^{n+n^{\prime}} \rightarrow \mathbb{R}^{n+n^{\prime}}$ defined by $\widetilde{f}(x, y)=(f(x), y)$ where $x \in \mathbb{R}^{n}$ and $y \in \mathbb{R}^{n^{\prime}}$.

It has been long believed that singularities of generic Riemannian exponential maps are generic Lagrangian singularities. This was stated by Weinstein [38] and confirmed in Wall [37. A complete proof appears in [23] (see also [24]).

More precisely we have the following result.

Theorem 3. Let $M$ be a smooth manifold with $\operatorname{dim} M \leq 5$, and fix $q \in M$. For generic Riemannian metrics on $M$, the singularities of the exponential map $\mathcal{E}_{q}$ are those listed in Theorem $\mathbb{Q}$.

A preliminary question to the study of the heat kernel asymptotics at generic singularities is to understand which singularities of the Arnol'd list can be obtained as "optimal" ones. In other words, we consider singularities of the exponential map that can arise along minimizing geodesics.

Definition 4. Let $M$ be a Riemannian manifold, $q_{1}, q_{2} \in M$ such that $\gamma(t)=\mathcal{E}_{q_{1}}(t v)$ for $0 \leq t \leq 1$ gives a minimizing conjugate geodesic from $q_{1}$ to $q_{2}$. Then we say that $\gamma$ is $A_{2}$-conjugate if at $v$, $\mathcal{E}_{q_{1}}$ has a normal form given by $A_{2}$. We define $A_{3}$-conjugacy, etc. in a similar way.

In Section 1 we prove the following result.

Theorem 5. Let $M$ be a smooth manifold, $\operatorname{dim} M \leq 5$, and $q_{1} \in M$. For a generic Riemannian metric on $M$ and any minimizing geodesic $\gamma$ from $q_{1}$ to some $q_{2}$ we have that $\gamma$ is either nonconjugate, $A_{3}$-conjugate, or $A_{5}$-conjugate (at $q_{2}$ ).

Notice that $A_{3}$ appears only for $n \geq 2$ and $A_{5}$ for $n \geq 4$. As a consequence of Theorem 5 and of Theorem 9 in Section 1.3 (which gives a finer analysis than that presented in [11] of the relation between the degree of conjugacy and the small-time heat kernel asymptotics), we get

Corollary 6. Let $M$ be a smooth manifold, $\operatorname{dim} M=n \leq 5$, and $q_{1} \in M$. For a generic Riemannian metric on $M$ the only possible heat kernel asymptotics are (here $C>0$ is some constant which can differ from line to line):

(i) If no minimizing geodesic from $q_{1}$ to $q_{2}$ is conjugate then

$$
p_{t}\left(q_{1}, q_{2}\right)=\frac{C+O(t)}{t^{\frac{n}{2}}} e^{-d^{2}\left(q_{1}, q_{2}\right) / 4 t}
$$

(ii) If at least one minimizing geodesic from $q_{1}$ to $q_{2}$ is $A_{3}$-conjugate but none is $A_{5}$-conjugate

$$
p_{t}\left(q_{1}, q_{2}\right)=\frac{C+O\left(t^{1 / 2}\right)}{t^{\frac{n}{2}+\frac{1}{4}}} e^{-d^{2}\left(q_{1}, q_{2}\right) / 4 t},
$$

(iii) If at least one minimizing geodesic from $q_{1}$ to $q_{2}$ is $A_{5}$-conjugate

$$
p_{t}\left(q_{1}, q_{2}\right)=\frac{C+O\left(t^{1 / 3}\right)}{t^{\frac{n}{2}+\frac{1}{6}}} e^{-d^{2}\left(q_{1}, q_{2}\right) / 4 t} .
$$

Notice that this is consistent with the results obtained in [12] on surfaces of revolution. 


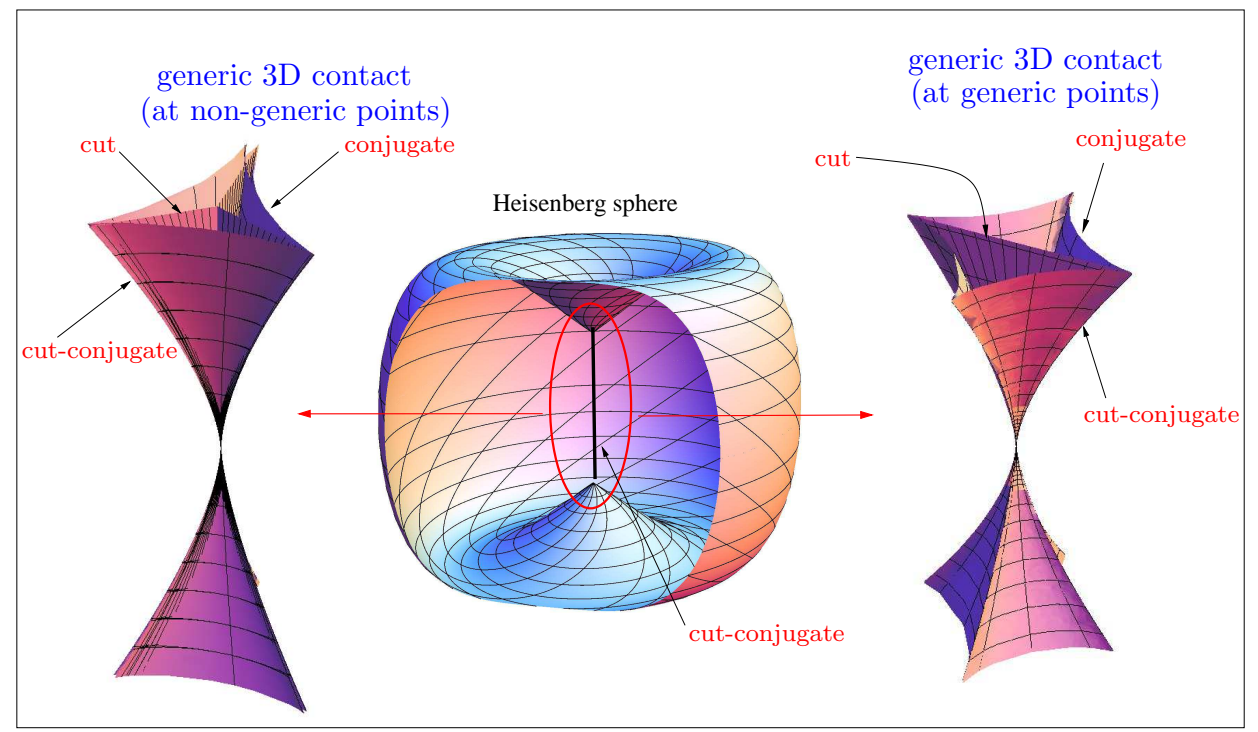

Figure 1: Cut locus and first conjugate locus in a generic 3D contact sub-Riemannian structure close to the starting point. The picture in the center shows the endpoints of optimal geodesics at time 1 for the Heisenberg group, which is the simplest 3D contact sub-Riemannian structure. The vertical axis is the cut locus, which coincides with the first conjugate locus. The picture on the right shows the cut locus and the first conjugate locus after a generic perturbation at a generic point. The first conjugate locus is a suspension of a four-cusp astroid. The picture on the left shows the cut locus and the first conjugate locus after a generic perturbation at a non-generic point. The first conjugate locus is a suspension of a "six-cusp astroid."

\subsection{The sub-Riemannian case}

Some of the techniques developed to prove Theorems 5 and Corollary 6 can be adapted to the sub-Riemannian context.

As in the Riemannian case, we always assume that the sub-Riemannian metrics are complete as metric spaces. Moreover, when we say generic, we always refer to open and dense subsets with respect to the $C^{\infty}$ Whitney topology (see Definition 1).

\subsubsection{D contact case}

In contact geometry, there are no nontrivial abnormal extremals (for the definition of abnormals see Remark 18). For the generic 3D contact case, in [6, 17] it is shown that close to the diagonal only singularities of type $A_{3}$ appear, accumulating to the initial point. The local structure of the first conjugate locus (see Fig. 1.2.1) is either a suspension of a four-cusp astroid (at generic points) or a suspension of a "six-cusp astroid" (along some special curves). For the four-cusp case, two of the cusps are reached by cut-conjugate geodesics, while in the six-cusp case this happens for three of them. Notice that the first conjugate locus at a generic point looks like a suspension of the first conjugate locus that one gets on a Riemannian ellipsoid [12]. The precise statement of these facts can be found in [6, 17] (see also [2]). 
As a consequence of this analysis and of Theorem 9 in Section 1.3 we get the following characterization of the heat kernel asymptotics for the 3D contact case.

Corollary 7. Let $M$ be a smooth manifold of dimension 3. Then for a generic 3D contact subRiemannian metric on $M$, every $q_{1}$, and every $q_{2}$ (close enough to $q_{1}$ ) we have that the only possible heat kernel asymptotics are (here $C>0$ is some constant which can differ from line to line):

(i) If no minimizing geodesic from $q_{1}$ to $q_{2}$ is conjugate then

$$
p_{t}\left(q_{1}, q_{2}\right)=\frac{C+O(t)}{t^{3 / 2}} e^{-d^{2}\left(q_{1}, q_{2}\right) / 4 t},
$$

(ii) If at least one minimizing geodesic from $q_{1}$ to $q_{2}$ is conjugate then

$$
p_{t}\left(q_{1}, q_{2}\right)=\frac{C+O\left(t^{1 / 2}\right)}{t^{7 / 4}} e^{-d^{2}\left(q_{1}, q_{2}\right) / 4 t} .
$$

Moreover, there are points $q_{2}$ arbitrarily close to $q_{1}$ such that case (ii) occurs.

Notice that exponents of the form $N / 4$, for integer $N$, were unexpected in the 90 s literature, see [27].

\subsubsection{D quasi-contact case}

For generic 4D quasi-contact metrics there are no non-trivial abnormal minimizers [5] (for nongeneric structures, nontrivial abnormal minimizers can exist but they are not strict). The structure of the local first conjugate locus is known for generic points, see [16]. More precisely, outside of a stratified set of codimension 1, the singularities corresponding to the first conjugate locus are of type $A_{2}, A_{3}$, and $D_{4}^{+}$. Moreover, among the points of the first conjugate locus, those that are cut-conjugate correspond to $A_{3}$ singularities, and they accumulate to the initial point.

Thanks to this analysis and to Theorem 9 (in Section 1.3) we get

Corollary 8. Let $M$ be a smooth manifold of dimension 4. Then, for a generic quasi-contact sub-Riemannian metric on $M$, for $q_{1}$ outside a stratified set of codimension 1 and $q_{2}$ close enough to $q_{1}$, the only possible heat kernel asymptotics are (here $C>0$ is some constant which can differ from line to line):

(i) If no minimizing geodesic from $q_{1}$ to $q_{2}$ is conjugate then

$$
p_{t}\left(q_{1}, q_{2}\right)=\frac{C+O(t)}{t^{2}} e^{-d^{2}\left(q_{1}, q_{2}\right) / 4 t},
$$

(ii) If at least one minimizing geodesic from $q_{1}$ to $q_{2}$ is conjugate then

$$
p_{t}\left(q_{1}, q_{2}\right)=\frac{C+O\left(t^{1 / 2}\right)}{t^{9 / 4}} e^{-d^{2}\left(q_{1}, q_{2}\right) / 4 t} .
$$

Moreover, there are points $q_{2}$ arbitrarily close to $q_{1}$ such that case (ii) occurs. 


\subsection{Techniques}

The analysis of the heat kernel asymptotics in the three cases of interest just discussed consists of a few steps. First, the structure of the minimizing geodesics must be understood. The possible generic singularities of the exponential map for the 3D contact and $4 \mathrm{D}$ quasi-contact cases were essentially already known, and we have already summarized those results. In the Riemannian case, this means that we wish to prove Theorem 5, which is of independent interest (in particular, compare the list of possible singularities occurring for minimizing geodesics with the much longer list of singularities that can occur for geodesics past the point of being minimizing, as given in Theorems 2 and 3). To do this, we consider the hinged energy function (see [30, 32, 11])

$$
h_{q_{1}, q_{2}}(q)=\frac{1}{2}\left(d^{2}\left(q_{1}, q\right)+d^{2}\left(q, q_{2}\right)\right) .
$$

This function achieves its minimum of $d^{2}\left(q_{1}, q_{2}\right) / 4$ precisely at the midpoints of minimizing geodesics from $q_{1}$ to $q_{2}$ [11, Lemma 21]. Further, the local structure of $h=h_{q_{1}, q_{2}}$ near these midpoints is closely related to the structure of the exponential map along the corresponding geodesic. In particular, because $h$ has a minimum at such a midpoint, its "normal form" is more restricted than at an arbitrary critical point (for example, in dimension two, $h$ cannot have a normal form of the type $x^{2}+y^{3}$ ). Transferring these restrictions from $h$ to the exponential map allows us to show that most of the singularities in Theorem 2 cannot occur at minimizing geodesics. The details are given in Section 3 .

Once we have a description of the possible singularities that can appear along a minimizing geodesic for a given generic geometry, we then determine the corresponding normal form for the singularity of $h$ at the midpoint of such a geodesic. Having determined the possible structures of $h$, we determine the corresponding heat-kernel asymptotics in the spirit of 11]. However we actually need a finer result than was given there.

This is of some independent interest, so we elaborate on it here. We will consider minimizing geodesics such that the exponential map has a singular point of type $(1, m)$ (see Definition 26); such geodesics include those which are $A_{m}$-conjugate. Here $h$ has the simple form $d^{2}\left(q_{1}, q_{2}\right) / 4+$

$x_{1}^{2}+\cdots+x_{n-1}^{2}+x_{n}^{m+1}$ with $m$ odd, in some coordinates around the midpoint of the geodesic (see Lemma 27). The corresponding heat kernel asymptotics are described in the following theorem, which gives a refinement of (part of) Theorem 27 from [11]. This also improves upon the pioneering work of Molchanov, see [30, Subcase 3.2.a].

Theorem 9. Let $M$ be an n-dimensional, complete (sub)-Riemannian manifold, and let $q_{1}$ and $q_{2}$ be distinct points such that all minimizing geodesics from $q_{1}$ to $q_{2}$ are strongly normal. Suppose that, for some $\ell \in\{3,5,7, \ldots\}$ every minimizing geodesic from $q_{1}$ to $q_{2}$ corresponds to a singular point of type $(1, m)$ with $3 \leq m \leq \ell$, and that there is at least one minimizing geodesics where the singular point is of type $(1, \ell)$. Then there exists $C>0$ such that

$$
p_{t}\left(q_{1}, q_{2}\right)=\frac{C+O\left(t^{\frac{2}{\ell+1}}\right)}{t^{\frac{n+1}{2}-\frac{1}{\ell+1}}} e^{-d^{2}\left(q_{1}, q_{2}\right) / 4 t} .
$$

Remark 10. Theorem 9 holds for (sub)-Riemannian structures as defined in Section 2, but also for more general rank-varying sub-Riemannian structures in the sense of [11, Appendix A]. 


\subsection{Complementary results and non-generic cases}

As a further refinement of Theorem 27 from [11], we also offer the following theorem (which, although not used elsewhere in the present paper, helps to shed additional light on the relationship between the structure of the minimizing geodesics and the small-time heat kernel asymptotics).

Recall that in the (sub)-Riemannian context, arclength geodesics starting from a point $q$ are parametrized by initial covectors $\lambda \in T_{q}^{*} M \cap\{H=1 / 2\}$ where $H$ is the (sub)-Riemannian Hamiltonian $H(q, \lambda)=\sum_{1}^{k}\left\langle\lambda, X_{i}(q)\right\rangle^{2}$. (Here $X_{1}, \ldots, X_{k}$ is an orthonormal frame for the structure.) Hence the exponential map $\mathcal{E}_{q}$ is a map that, with $\lambda \in T_{q}^{*} M \cap\{H=1 / 2\}$ and a time $t$, associates the end point $\mathcal{E}_{q}(t \lambda)$ of the arclength parameterised geodesic defined on $[0, t]$, starting at $q$, and corresponding to $\lambda$. Recall that $\mathcal{E}_{q}$ depends only on the product $t \lambda$ and not on $t$ and $\lambda$ separately. Hence $\mathcal{E}_{q}$ can be thought as defined on the whole of $T_{q}^{*} M$.

First, suppose that $q_{1}$ and $q_{2}$ are distinct points on $M$, and let $\lambda \in T_{q_{1}}^{*} M$ be such that $\mathcal{E}_{q_{1}}(2 t \lambda)$ for $0 \leq t \leq 1$ gives a minimizing geodesic from $q_{1}$ to $q_{2}$. Then recall from Theorem 24 of [11] that $d \mathcal{E}_{q_{1}}$ at $2 \lambda$ has rank $n-r$ if and only if the Hessian of $h_{q_{1}, q_{2}}$ at $\mathcal{E}_{q_{1}}(\lambda)$ has rank $n-r$. The idea is that this rank partially describes how conjugate this minimizing geodesic is by indicating the number of independent perturbations with respect to which it is conjugate.

Theorem 11. Let $M$ be an n-dimensional complete (sub)-Riemannian manifold, and let $q_{1}$ and $q_{2}$ be distinct points such that there is a unique minimizing strongly normal geodesic from $q_{1}$ to $q_{2}$ (which we denote $\mathcal{E}_{q_{1}}(2 t \lambda)$ for $0 \leq t \leq 1$, as above). Then if $D_{2 \lambda} \mathcal{E}_{q_{1}}$ has rank $n-r$ for some $r \in\{0,1,2, \ldots, n-1\}$, there exist $C_{1}, C_{2}, t_{0}>0$ such that for $0<t<t_{0}$

$$
\frac{C_{1}}{t^{\frac{n}{2}+\frac{r}{4}}} e^{-d^{2}\left(q_{1}, q_{2}\right) / 4 t} \leq p_{t}\left(q_{1}, q_{2}\right) \leq \frac{C_{2}}{t^{\frac{n}{2}+\frac{r}{2}}} e^{-d^{2}\left(q_{1}, q_{2}\right) / 4 t} .
$$

Remark 12. As for Theorem 9, Theorem 11 holds for (sub)-Riemannian structures as defined in Section 2, but also for more general rank-varying sub-Riemannian structures in the sense of [11, Appendix A].

The previous two theorems (namely Theorem 9 and Theorem 11) present an especially appealing picture when considered together. Simply knowing the rank of the exponential map, even when there is only a single minimizing geodesic, does not completely determine the asymptotic behavior. Instead, the precise behavior in the degenerate directions matters, as Theorem 9 indicates. Indeed, both $A_{3}$ and $A_{5}$-conjugacy fall into the $r=1$ case of Theorem 11. Further, one could consider the contribution to the small-time asymptotics of minimizing geodesics exhibiting other singularities from the Arnol'd classification (Theorem 9 dealing with the case when the only singularities are from the $A_{m}$-family), and determine which ones dominate which other ones. However, the situation becomes more complicated as more types of singularities are allowed. Indeed, the virtue of the lowdimensional cases considered here is that the list of possible singularities which can occur, at least generically, is sufficiently simple so as to allow a complete analysis.

On the other hand, if one is willing to go beyond the generic case, a wide variety of expansions are possible, even for Riemannian metrics in low dimensions. To illustrate this, we give the following theorem, highlighting the contrast between the generic and non-generic situations (compare this with Corollary (6).

Theorem 13. For any integer $\eta \geq 3$, any positive real $\alpha$, and any real $\beta$, there exists a smooth metric on the sphere $\mathbb{S}^{2}$ and (distinct) points $q_{1}$ and $q_{2}$ such that the heat kernel has the small-time 
asymptotic expansion

$$
p_{t}\left(q_{1}, q_{2}\right)=e^{-d^{2}\left(q_{1}, q_{2}\right) / 4 t} \frac{1}{t^{(3 \eta-1) / 2 \eta}}\left\{\alpha+t^{1 / \eta} \beta+o\left(t^{1 / \eta}\right)\right\} .
$$

Note that both the leading term and the next term in this expansion are precisely what one gets by applying Theorem 9 to a surface and letting $\ell=2 \eta-1$. Thus, the existence of such expansions is not surprising. Nonetheless, the point of Theorem 13 is to show that such singularities do in fact occur (in the proof of Theorem 13, we will see that the metric we construct does yield a singularity of type $(1, m)$ for appropriate $m)$, and further, that the "big-O" term we see for the next term in the expansion cannot in general be improved upon. That is, we do see expansions in fractional powers of $t$, as Theorem 9 seems to suggest.

\section{2 (Sub)-Riemannian geometry}

We start by recalling the definition of a (sub)-Riemannian manifold (including also Riemannian manifolds) in the case of a distribution of constant rank.

Definition 14. A (sub)-Riemannian manifold is a triple $(M, \mathcal{D}, g)$, where

(i) $M$ is a connected, orientable, smooth manifold of dimension $n$.

(ii) $\mathcal{D}$ is a smooth vector distribution of constant rank $k \leq n$ satisfying the Hörmander condition, that is, a smooth map that associates with $q \in M$ a $k$-dimensional subspace $\mathcal{D}_{q}$ of $T_{q} M$ such that

$$
\operatorname{span}\left\{\left[X_{1},\left[\ldots\left[X_{j-1}, X_{j}\right]\right]\right]_{q} \mid X_{i} \in \overline{\mathcal{D}}, j \in \mathbb{N}\right\}=T_{q} M, \forall q \in M,
$$

where $\overline{\mathcal{D}}$ denotes the set of horizontal smooth vector fields on $M$, i.e.

$$
\overline{\mathcal{D}}=\left\{X \in \operatorname{Vec}(M) \mid X(q) \in \mathcal{D}_{q} \forall q \in M\right\} .
$$

(iii) $g_{q}$ is a Riemannian metric on $\mathcal{D}_{q}$ which is smooth as function of $q$. We denote the norm of a vector $v \in \mathcal{D}_{q}$ by $|v|_{g}=\sqrt{g_{q}(v, v)}$.

Remark 15. Notice that, when $k=n$, we are in the Riemannian context and the Hörmander condition is automatically satisfied. As a consequence of the definition, (sub)-Riemannian structures that are not Riemannian exist only in dimension $n \geq 3$.

A Lipschitz continuous curve $\gamma:[0, T] \rightarrow M$ is said to be horizontal (or admissible) if

$$
\dot{\gamma}(t) \in \mathcal{D}_{\gamma(t)} \quad \text { for a.e. } t \in[0, T] .
$$

Given a horizontal curve $\gamma:[0, T] \rightarrow M$, the length of $\gamma$ is

$$
\ell(\gamma)=\int_{0}^{T}|\dot{\gamma}(t)|_{g} d t
$$

Notice that $\ell(\gamma)$ is invariant under time reparametrization of the curve $\gamma$. The distance induced by the (sub)-Riemannian structure on $M$ is the function

$$
d\left(q_{0}, q_{1}\right)=\inf \left\{\ell(\gamma) \mid \gamma(0)=q_{0}, \gamma(T)=q_{1}, \gamma \text { horizontal }\right\}
$$


The hypothesis of connectedness of $M$ and the Hörmander condition guarantee the finiteness and the continuity of $d(\cdot, \cdot)$ with respect to the topology of $M$ (Chow-Rashevsky theorem, see for instance [8] $)$. The function $d(\cdot, \cdot)$ is called the Carnot-Caratheodory distance and gives to $M$ the structure of a metric space (see for instance [2]).

Locally, the pair $(\mathcal{D}, g)$ can be given by assigning a set of $k$ smooth vector fields that span $\mathcal{D}$ and that are orthonormal for $g$, i.e.

$$
\mathcal{D}_{q}=\operatorname{span}\left\{X_{1}(q), \ldots, X_{k}(q)\right\}, \quad g_{q}\left(X_{i}(q), X_{j}(q)\right)=\delta_{i j} .
$$

In this case, the set $\left\{X_{1}, \ldots, X_{k}\right\}$ is called a local orthonormal frame for the sub-Riemannian structure.

A sub-Riemannian manifold of odd dimension is said to be contact if $\mathcal{D}=\operatorname{ker} \omega$, where $\omega \in \Lambda^{1} M$ and $\left.d \omega\right|_{\mathcal{D}}$ is non degenerate. A sub-Riemannian manifold $M$ of even dimension is said to be quasicontact if $\mathcal{D}=\operatorname{ker} \omega$, where $\omega \in \Lambda^{1} M$ and satisfies $\left.\operatorname{dim} \operatorname{ker} d \omega\right|_{\mathcal{D}}=1$.

The sub-Riemannian metric can also be expressed locally in "control form" as follows. We consider the control system,

$$
\dot{q}=\sum_{i=1}^{m} u_{i} X_{i}(q), \quad u_{i} \in \mathbb{R}
$$

and the problem of finding the shortest curve that joins two fixed points $q_{0}, q_{1} \in M$ is naturally formulated as the optimal control problem

$$
\int_{0}^{T} \sqrt{\sum_{i=1}^{m} u_{i}^{2}(t)} d t \rightarrow \min , \quad q(0)=q_{0}, \quad q(T)=q_{1} \neq q_{0} .
$$

\subsection{Minimizers and geodesics}

In this section we briefly recall some facts about (sub)-Riemannian geodesics. In particular, we define the (sub)-Riemannian exponential map.

Definition 16. A geodesic for a (sub)-Riemannian manifold $(M, \mathcal{D}, g)$ is an admissible curve $\gamma$ : $[0, T] \rightarrow M$ such that $|\dot{\gamma}(t)|_{g}$ is constant and, for every sufficiently small interval $\left[t_{1}, t_{2}\right] \subset[0, T]$, the restriction $\gamma_{\left[t_{1}, t_{2}\right]}$ is a minimizer of $\ell(\cdot)$. A geodesic for which $|\dot{\gamma}(t)|_{g}=1$ is said to be parametrized by arclength. A (sub)-Riemannian manifold is said to be complete if $(M, d)$ is complete as a metric space.

Notice that, if the (sub)-Riemannian metric is the restriction to $\mathcal{D}$ of a complete Riemannian metric, then it is complete. Under the assumption that the manifold is complete, a version of the Hopf-Rinow theorem (see [36] or [15, Chapter 2]) implies that the manifold is geodesically complete (i.e. all geodesics are defined for every $t \geq 0$ ) and that for every two points there exists a minimizing geodesic connecting them.

Trajectories minimizing the distance between two points are solutions of first-order necessary conditions for optimality, which in the case of (sub)-Riemannian geometry are given by a weak version of the Pontryagin Maximum Principle (34]). 
Theorem 17. Let $q(\cdot): t \in[0, T] \mapsto q(t) \in M$ be a solution of the minimization problem (16),(7) such that $|\dot{q}(t)|_{g}$ is constant and $u(\cdot)$ be the corresponding control. Then there exists a Lipschitz map $p(\cdot): t \in[0, T] \mapsto p(t) \in T_{q(t)}^{*} M \backslash\{0\}$ such that one and only one of the following conditions holds:

(i) $\dot{q}=\frac{\partial H}{\partial p}, \quad \dot{p}=-\frac{\partial H}{\partial q}, \quad u_{i}(t)=\left\langle p(t), X_{i}(q(t))\right\rangle$, where $H(q, p)=\frac{1}{2} \sum_{i=1}^{k}\left\langle p, X_{i}(q)\right\rangle^{2}$.

(ii) $\dot{q}=\frac{\partial \mathcal{H}}{\partial p}, \quad \dot{p}=-\frac{\partial \mathcal{H}}{\partial q}, \quad 0=\left\langle p(t), X_{i}(q(t))\right\rangle$ where $\mathcal{H}(t, q, p)=\sum_{i=1}^{k} u_{i}(t)\left\langle p, X_{i}(q)\right\rangle$.

For an elementary proof of Theorem 17 see [2].

Remark 18. If $(q(\cdot), p(\cdot))$ is a solution of (i) (resp. (ii)) then it is called a normal extremal (resp. abnormal extremal). It is well known that if $(q(\cdot), p(\cdot))$ is a normal extremal then $q(\cdot)$ is a geodesic (see [2, 8]). This does not hold in general for abnormal extremals. An admissible trajectory $q(\cdot)$ can be at the same time normal and abnormal (corresponding to different covectors). If an admissible trajectory $q(\cdot)$ is normal but not abnormal, we say that it is strictly normal. Abnormal extremals do not exist in the Riemannian case. Abnormal extremals are very difficult to treat and many questions are still open. For instance, it is not known if abnormal minimizers are smooth (see [31]).

Definition 19. A minimizer $\gamma:[0, T] \rightarrow M$ is said to be strongly normal if for every $\left[t_{1}, t_{2}\right] \subset[0, T]$, $\left.\gamma\right|_{\left[t_{1}, t_{2}\right]}$ is not an abnormal.

In the following, we denote by $(q(t), p(t))=e^{t \vec{H}}\left(q_{0}, p_{0}\right)$ the solution of (i) of Theorem 17 with initial condition $(q(0), p(0))=\left(q_{0}, p_{0}\right)$. Moreover we denote by $\pi: T^{*} M \rightarrow M$ the canonical projection.

Normal extremals (starting from $q_{0}$ ) parametrized by arclength correspond to initial covectors $p_{0} \in \Lambda_{q_{0}}:=\left\{p_{0} \in T_{q_{0}}^{*} M \mid H\left(q_{0}, p_{0}\right)=1 / 2\right\}$. The exponential map starting from $q_{0}$ is defined as

$$
\mathcal{E}_{q_{0}}: \mathbb{R}^{+} \times \Lambda_{q_{0}} \rightarrow M, \quad \mathcal{E}_{q_{0}}\left(t p_{0}\right)=\pi\left(e^{t \vec{H}}\left(q_{0}, p_{0}\right)\right) .
$$

Next, we recall the definition of cut and first conjugate times.

Definition 20. Let $q_{0} \in M$ and $\gamma(t)$ an arclength geodesic starting from $q_{0}$. The cut time for $\gamma$ is $t_{\text {cut }}(\gamma)=\sup \left\{t>0:\left.\gamma\right|_{[0, t]}\right.$ is optimal $\}$. The cut locus from $q_{0}$ is the set $\operatorname{Cut}\left(q_{0}\right)=\left\{\gamma\left(t_{\text {cut }}(\gamma)\right): \gamma\right.$ is an arclength geodesic from $\left.q_{0}\right\}$.

Definition 21. Let $q_{0} \in M$ and $\gamma(t)$ a normal arclength geodesic starting from $q_{0}$ with initial covector $p_{0}$. Assume that $\gamma$ is not abnormal. The first conjugate time of $\gamma$ is $t_{\text {con }}(\gamma)=\min \{t>0$ : $\left(t, p_{0}\right)$ is a critical point of $\left.\mathcal{E}_{q_{0}}\right\}$. The first conjugate locus from $q_{0}$ is the set $\operatorname{Con}\left(q_{0}\right)=\left\{\gamma\left(t_{\text {con }}(\gamma)\right): \gamma\right.$ is an arclength geodesic from $\left.q_{0}\right\}$.

It is well known that, for a geodesic $\gamma$ which is not abnormal, the cut time $t_{*}=t_{\text {cut }}(\gamma)$ is either equal to the conjugate time or there exists another geodesic $\widetilde{\gamma}$ such that $\gamma\left(t_{*}\right)=\widetilde{\gamma}\left(t_{*}\right)$ (see for instance [6]).

Remark 22. For sub-Riemannian manifolds (that are not Riemannian), the exponential map starting from $q_{0}$ is never a local diffeomorphism in a neighborhood of the point $q_{0}$ itself. As a consequence the sub-Riemannian balls are never smooth and both the cut and the conjugate loci from $q_{0}$ are adjacent to the point $q_{0}$ itself (see [1]). 


\subsection{The (sub)-Laplacian}

In this section we define the (sub)-Riemannian Laplacian on a (sub)-Riemannian manifold $(M, \mathcal{D}, g)$, provided with a smooth volume $\mu$. The (sub)-Laplacian is the natural generalization of the LaplaceBeltrami operator on a Riemannian manifold, defined as the divergence of the (horizontal) gradient (see for instance [4]). In a local orthonormal frame $X_{1}, \ldots, X_{k}$ it can be written as follows

$$
\Delta=\sum_{i=1}^{k} X_{i}^{2}+\left(\operatorname{div} X_{i}\right) X_{i}
$$

Notice that $\Delta$ is always expressed as the sum of squares of the elements of the orthonormal frame, plus a first-order term that belongs to the distribution and depends on the choice of the volume $\mu$.

Thanks to the Hörmander condition, the sub-Laplacian is hypoelliptic [21]. The existence of a smooth heat kernel for the operator $\Delta$, in the case of a complete sub-Riemannian manifold, is stated in 36].

Remark 23. We recall that in the equiregular case, it is possible to define in an intrinsic way a volume form on the (sub)-Riemannian manifold (called Popp's volume [31], which coincides with the Riemannian volume in the Riemannian case). When the (sub)-Laplacian is computed with respect to Popp's volume it is called the "intrinsic-Laplacian". Other intrinsic non-equivalent volumes can be defined in certain cases, see [3].

\section{Technical lemmas}

We first recall two technical results that we need. The first one is the "splitting lemma" for smooth functions (which can be found in [20]) which allows us to split off non-degenerate directions and thus partially diagonalize $g$.

Lemma 24 ([20]). Let $g: U \subset \mathbb{R}^{n}$ be a smooth function on a neighborhood $U$ of the origin in $\mathbb{R}^{n}$. Assume that $g(0)=d g(0)=0$ and that 0 is an isolated local minimum of $g$ with dim $\operatorname{ker} d^{2} g(0)=k$.

Then there exists a diffeomorphism $\psi$ from a neighborhood of the origin in $\mathbb{R}^{n}$ to a neighborhood of the origin in $\mathbb{R}^{n}$ and a smooth function $\varphi: \mathbb{R}^{k} \rightarrow \mathbb{R}$ such that

$$
g(\psi(u))=u_{1}^{2}+\ldots+u_{n-k}^{2}+\varphi\left(u_{n-k+1}, \ldots, u_{n}\right) .
$$

The second result relates the structure of minimizing geodesics from $q_{1}$ to $q_{2}$ to the behaviour of $h_{q_{1}, q_{2}}$ near its minimum. Suppose we have distinct points $q_{1}$ and $q_{2}$ such that every minimizer from $q_{1}$ to $q_{2}$ is strongly normal. Let $\Gamma$ be the set of midpoints of minimizing geodesics from $q_{1}$ to $q_{2}$.

Consider any point $z_{0} \in \Gamma$, which is the midpoint of some minimizing geodesic $\gamma$ from $q_{1}$ to $q_{2}$. Then there is a unique covector $\lambda \in T_{q_{1}}^{*} M$ such that $\mathcal{E}_{q_{1}}(\lambda)=z_{0}, \mathcal{E}_{q_{1}}(2 \lambda)=q_{2}$ and that $\mathcal{E}_{q_{1}}(2 t \lambda)$ for $t \in[0,1]$ parametrizes $\gamma$.

Let $\lambda(s)$ be a smooth curve of covectors $\lambda:(-\varepsilon, \varepsilon) \rightarrow T_{q_{1}}^{*} M$ (for some small $\varepsilon>0$ ) such that $\lambda(0)=\lambda$ and the derivative never vanishes. Thus $\lambda(s)$ is a one-parameter family of perturbations of $\lambda$ which realizes the first-order perturbation $\lambda^{\prime}(0) \in T_{\lambda}\left(T_{q_{1}}^{*} M\right)$. Also, we let $z(s)=\mathcal{E}_{q_{1}}(\lambda(s))$, so that $z(0)=z_{0}$. Because $\mathcal{E}_{q_{1}}$ is a diffeomorphism from a neighborhood of $\lambda$ to a neighborhood of $z_{0}$, we see that the derivative of $z(s)$ also never vanishes. Thus $z(s)$ is a curve which realizes the 
vector $z^{\prime}(0) \in T_{z_{0}} M$. Further, we've established an isomorphism of the vector spaces $T_{\lambda}\left(T_{q_{1}}^{*} M\right)$ and $T_{z_{0}} M$ by mapping $\lambda^{\prime}(0)$ to $z^{\prime}(0)$, except that we've excluded the origin by insisting that both vectors are non-zero.

We say that $\gamma$ is conjugate in the direction $\lambda^{\prime}(0)$ (or with respect to the perturbation $\lambda^{\prime}(0)$ ) if $\left.\frac{d}{d s} \mathcal{E}_{q_{1}}(2 \lambda(s))\right|_{s=0}=0$. Note that this only depends on $\lambda^{\prime}(0)$. We say that the Hessian of $h_{q_{1}, q_{2}}$ at $z_{0}$ is degenerate in the direction $z^{\prime}(0)$ if $\left.\frac{d^{2}}{d s^{2}} h_{q_{1}, q_{2}}(z(s))\right|_{s=0}=0$. This last equality is equivalent to writing the Hessian of $h_{q_{1}, q_{2}}$ as a matrix in some smooth local coordinates, applying it as a quadratic form to $z^{\prime}(0)$ expressed in these coordinates, and getting zero. This equivalence, as well as the fact that whether the result is zero or not depends only on $z^{\prime}(0)$, follows from the fact that $z_{0}$ is a critical point of $h_{q_{1}, q_{2}}$.

The point of the next theorem is that conjugacy in the direction $\lambda^{\prime}(0)$ is equivalent to degeneracy in the direction $z^{\prime}(0)$.

Theorem 25 (11]). Let $M$ be a complete (sub)-Riemannian manifold, and let $q_{1}$ and $q_{2}$ be distinct points such that every minimizing geodesic from $q_{1}$ to $q_{2}$ is strongly normal. Let $\gamma$ be a minimizing geodesic from $q_{1}$ to $q_{2}$, and define $\Gamma, z_{0} \in \Gamma, h_{q_{1}, q_{2}}$ and the curves $\lambda(s), z(s)$ as above. Then

(i) $\gamma$ is conjugate if and only if the Hessian of $h_{q_{1}, q_{2}}$ at $z_{0}$ is degenerate.

(ii) In particular $\gamma$ is conjugate in the direction $\lambda^{\prime}(0)$ if and only if the Hessian of $h_{q_{1}, q_{2}}$ at $z_{0}$ is degenerate in the corresponding direction $z^{\prime}(0)$.

(iii) The dimension of the kernel of $D_{2 \lambda} \mathcal{E}_{q_{1}}$ is equal to the dimension of the kernel of the Hessian of $h_{q_{1}, q_{2}}$ at $z_{0}$.

We now need the following

Definition 26. Let $f: M \rightarrow N$ be a smooth map between two $n$-dimensional manifolds and $q \in M$. We say that $q$ is a singular point of type $(1, m)$ for $f$ if

(i) $\operatorname{rank}\left(D_{q} f\right)=n-1$.

(ii) in a system of coordinates we have

$$
m=\max \left\{k \in \mathbb{N} \mid f(\gamma(t))=f(q)+t^{k} v+o\left(t^{k}\right), v \neq 0, \gamma \in \mathcal{C}_{q}\right\}
$$

where $\mathcal{C}_{q}$ is the set of smooth curves $\gamma$ such that $\gamma(0)=q$ and $\gamma^{\prime}(0) \neq 0$.

Notice that in this definition one necessarily has $m \geq 2$. The next two lemmas are crucial in what follows.

Lemma 27. Let $q_{1}$ and $q_{2}$ be conjugate along a minimizing geodesic $\gamma(t)=\mathcal{E}_{q_{1}}(2 t \lambda)$ for $t \in[0,1]$, and let $z_{0}$ be the midpoint. Then $2 \lambda$ is a singular point of type $(1, m)$ for $\mathcal{E}_{q_{1}}$ if and only if there exists a system of coordinates $\left(x_{1}, \ldots, x_{n}\right)$ around $z_{0}$ such that

$$
h_{q_{1}, q_{2}}(x)=\frac{1}{4} d^{2}\left(q_{1}, q_{2}\right)+x_{1}^{2}+\ldots+x_{n-1}^{2}+x_{n}^{m+1} .
$$


Proof. We prove only that if $2 \lambda$ is a singular point of type $(1, m)$ for $\mathcal{E}_{q_{1}}$ then there exists coordinates such that (10) holds. The converse is similar.

Thanks to Theorem 25, we have that dim $\operatorname{ker} D_{2 \lambda} \mathcal{E}_{q_{1}}=1$ if and only dim ker Hess $z_{z_{0}} h_{q_{1}, q_{2}}=1$. Hence, by Lemma 24, there exists a coordinate system at $z_{0}=\mathcal{E}_{q_{1}}(\lambda)$ such that $h_{q_{1}, q_{2}}\left(x_{1}, \ldots, x_{n}\right)=$ $h\left(z_{0}\right)+x_{1}^{2}+\cdots+x_{n-1}^{2}+\varphi\left(x_{n}\right)$ with $\varphi$ smooth. Since $h\left(z_{0}\right)=d^{2}\left(q_{1}, q_{2}\right) / 4$, we are left to check that we can choose the coordinate system in such a way that $\varphi\left(x_{n}\right)=x_{n}^{m+1}$.

Consider any smooth curve $s \mapsto \lambda(s)$ in $T_{q_{1}}^{*} M$ such that $\lambda(0)=\lambda$, and write $x(s)=\mathcal{E}_{q_{1}}(\lambda(s))$ and $y(s)=\mathcal{E}_{q_{1}}(2 \lambda(s))$. It is clear that $x(0)=z_{0}$ and $y(0)=q_{2}$. Using that $z_{0}$, the midpoint of the curve, does not belong to the cut locus of $q_{2}$, one can prove (see the proof of Theorem 25 in [11]) that the families $d_{x(s)} h_{q_{1}, q_{2}}$ and $y(s)-q_{2}$ have the same order with respect to $s$ (in any coordinate system). In particular, if we choose the family $\lambda(s)$ corresponding to the curve $x(s)=(0, \ldots, 0, s)$ (this is possible since the exponential map is a diffeomorphism near these points), then $d_{(0, \ldots, 0, s)} h_{q_{1}, q_{2}}=\varphi^{\prime}(s) d x_{n}$. Now assume that $\varphi\left(x_{n}\right)=c x_{n}^{k+1}+o\left(x_{n}^{k+1}\right)$ with $c \neq 0$. Up to a reparameterization of the $x_{n}$-axis, we can assume that $\varphi\left(x_{n}\right)=x_{n}^{k+1}$. We want to show that $k=m$. Indeed it is easy to show that for every choice of a curve $\lambda(s)$ as before, $d_{x(s)} h_{q_{1}, q_{2}}$ has an order less or equal to $k$ which implies that $y(s)-q_{2}$ has order not greater than $k$. Hence $k$ is maximal and coincides with $m$.

Lemma 28. Let $q_{1}$ and $q_{2}$ be conjugate along a minimizing geodesic $\gamma(t)=\mathcal{E}_{q_{1}}(2 t \lambda)$ for $t \in[0,1]$. For any $\xi \in \operatorname{ker} D_{2 \lambda} \mathcal{E}_{q_{1}}$ exists a curve $s \mapsto \lambda(s)$ with $\lambda(0)=\lambda, \dot{\lambda}(0)=\xi$ and such that if $y(s)=$ $\mathcal{E}_{q_{1}}(2 \lambda(s))$ then in coordinates $q_{2}-y(s)=O\left(s^{3}\right)$.

Proof. The proof is similar to that of Lemma 27. Assume that $\operatorname{dim} \operatorname{ker} D_{2 \lambda} \mathcal{E}_{q_{1}}=k$, and let $z_{0}$ be the midpoint of $\gamma$. Then also dim ker $\operatorname{Hess}_{z_{0}} h_{q_{1}, q_{2}}=k$ and by Lemma 24 there exists a system of coordinates $\left(x_{1}, \ldots, x_{n-k}, \ldots, x_{n}\right)$ around $z_{0}$ such that $h_{q_{1}, q_{2}}\left(x_{1}, \ldots, x_{n}\right)=h\left(z_{0}\right)+$ $x_{1}^{2}+\cdots+x_{n-k}^{2}+\varphi\left(x_{n-k+1}, \ldots, x_{n}\right)$ where $\varphi$ is a smooth function. Moreover $\operatorname{ker} \operatorname{Hess}_{z_{0}} h_{q_{1}, q_{2}}=$ $\operatorname{span}\left\{\partial_{x_{n-k+1}}, \ldots, \partial_{x_{n}}\right\}$. This implies in particular that the Taylor series of $\varphi$ of order 2 is zero. In addition, since $z_{0}$ is a minimum of $h_{q_{1}, q_{2}}$, the Taylor series of $\varphi$ of order 3 is also zero at this point. (See for instance the proof of Lemma 30.) As a consequence, the differential $d \varphi$ has Taylor series of order 2 that vanishes at the origin.

Let now $w=\left(w_{1}, \ldots, w_{n}\right) \in \operatorname{ker~Hess}_{z_{0}} h_{q_{1}, q_{2}}$. It satisfies $w_{1}=\cdots=w_{n-k}=0$. Let $z(\cdot)$ be defined by $z(s)=w s$ and $\lambda(\cdot)$ be the corresponding curve of covectors in $T_{q_{1}}^{*} M$. If we define $y(s)=\mathcal{E}_{q_{1}}(2 \lambda(s))$, then $y(s)-q_{2}$ has vanishing Taylor series of order 2 (indeed it has the same order as $\left.d_{z(s)} h_{q_{1}, q_{2}}=\left.d \varphi\right|_{\left(x_{n-k+1}(s), \ldots, x_{n}(s)\right)}\right)$. Now, since the set of vectors in the kernel of the Hessian of $h_{q_{1}, q_{2}}$ is in bijection with the set of vectors in the kernel of $D_{2 \lambda} \mathcal{E}_{q_{1}}$, the lemma is proved.

\section{Proof of Theorem 5}

The idea of the proof is to identify, among the singularities that appear in the Arnol'd classification, only those that are reached by optimal geodesics. To this end, we will exploit the necessary conditions given by Lemmas 27 and 28 .

Lemma 29. Singularities $A_{m}$ are of type $(1, m)$, in the sense of Definition 20. 
Proof. From the normal form of $A_{m}$, that can be rewritten as

$$
\Psi:\left(x, y_{1}, \ldots, y_{m-2}\right) \mapsto\left(x^{m}+\sum_{i=1}^{m-2} x^{i} y_{i}, y_{1}, \ldots, y_{m-2}\right),
$$

it is clear that $A_{m}$ is a singularity of corank 1, that is, the dimension of the kernel of the differential at zero is 1 and is spanned by $\partial_{x}$. Moreover, the image of the curve $\gamma(s)=(s, 0, \ldots, 0)$ under $\Psi$ is $\Psi(\gamma(s))=\left(s^{m}, \ldots, 0\right)$. Hence there exists a non-singular curve starting from the origin such that its image has order $m$ in the variable $s$. Let us show that there exists no curve $\gamma(s)$ such that $\Psi(\gamma(s))$ has order bigger than $m$. We can assume that $\dot{\gamma}(0)=\partial_{x}$ (otherwise $\dot{\gamma}(0)$ is not in the kernel of $D_{0} \Psi$ and the image $\Psi(\gamma(t))$ has order 1$)$. It follows that $x(s) \sim s$. Assume now that $\Psi(\gamma(s))$ has order bigger than or equal to $m$. Then $y_{i}(s)$ has order bigger or equal to $m$ for every $i$. Thus the first coordinate of the image of $\Psi(\gamma(s))$, namely $x(s)^{m}+\sum_{i} x(s)^{i} y_{i}(s)$, has order exactly $m$. Hence there is no non-singular curve passing through the origin, the image of which has a degree higher than $m$.

From Lemma 29 and Lemma 27, it follows immediately that $A_{m}$ can be optimal if and only if $m$ is odd. Let us show now that all other singularities of the Arnol'd list can not appear as optimal singularities. Indeed it is enough to show that these singularities do not satisfy the conclusion of Lemma 28. We give a proof for the $D_{4}^{+}$and $E_{6}^{-}$cases. The other cases can be treated in a similar way.

$\left(D_{4}^{+}\right)$Let us consider the singularity $D_{4}^{+}$which has normal form

$$
\Psi:(x, y, z) \mapsto\left(x^{2}+y^{2}+x z, x y, z\right) .
$$

The kernel of the differential of $\Psi$ at zero is given by $\operatorname{span}\left\{\partial_{x}, \partial_{y}\right\}$. In particular, let us show that there exists no curve $\gamma(t)$ such that $\dot{\gamma}(0)=\partial_{x}$ and such that $\Psi(\gamma(t))$ has order greater than or equal to 3. Indeed we can assume $\gamma(t)=\left(a t+O\left(t^{2}\right), O\left(t^{2}\right), O\left(t^{2}\right)\right)$ where $a \neq 0$. Thus $\Psi(\gamma(t))=\left(a^{2} t^{2}+O\left(t^{3}\right), O\left(t^{3}\right), O\left(t^{2}\right)\right)$ which has always order 2.

$\left(E_{6}^{-}\right)$The singularity $E_{6}^{-}$has normal form

$$
\Psi:(x, y, z, t, u) \mapsto\left(x^{2}+x y z+t y+u x,-y^{3}+x^{2} z+t x, z, t, u\right) .
$$

The kernel of the differential of $\Psi$ at zero is given by $\operatorname{span}\left\{\partial_{x}, \partial_{y}\right\}$. Let us consider a curve $\gamma(t)$ such that $\dot{\gamma}(0)=\partial_{x}$. Thus we can assume, as before, that $\gamma(t)=\left(a t+O\left(t^{2}\right), O\left(t^{2}\right), \ldots, O\left(t^{2}\right)\right)$, with $a \neq 0$. Again, it is easy to see that $\Psi(\gamma(t))=\left(a^{2} t^{2}+O\left(t^{3}\right), O\left(t^{2}\right), \ldots, O\left(t^{2}\right)\right)$ which is of order 2 .

\section{Proof of Theorem 9 and of Corollaries [6, 7, 8]}

Suppose that $D$ is a compact set of $\mathbb{R}^{n}$ having the origin in its interior and that $f$ is smooth in a neighborhood of $D$. With $x=\left(x_{1}, \ldots, x_{n}\right)$, let

$$
g(x)=g(0)+\sum_{i=1}^{n} x_{i}^{2 m_{i}}
$$


for some integers $1 \leq m_{1} \leq m_{2} \leq \cdots \leq m_{n}$. Further, we let $\ell \in\{1, \ldots, n\}$ be the smallest integer such that $m_{\ell}=m_{n}$. For functions $g$ of this form, arbitrarily many terms of the asymptotics of the corresponding Laplace integral are known (as developed in the book of Kanwal and Estrada [18], for example). For our purposes, we will need only the first two terms. In particular, we have the following asymptotic expansion as $t \searrow 0$,

$$
\begin{aligned}
& \int_{D} f(x) e^{-g(x) / t} d x_{1} \cdots d x_{n}=\exp [-g(0) / t] t^{\frac{1}{2 m_{1}}+\cdots+\frac{1}{2 m_{n}} \times} \\
& \quad\left\{\left[\prod_{i=1}^{n} \frac{\Gamma\left(1 / 2 m_{i}\right)}{m_{i}}\right] f(0)+t^{1 / m_{n}}\left[\frac{\Gamma\left(3 / 2 m_{n}\right)}{2} \prod_{i=1}^{n-1} \frac{\Gamma\left(1 / 2 m_{i}\right)}{m_{i}}\right]\left[\sum_{\xi=\ell}^{n} \frac{\partial^{2} f}{\partial x_{\xi}^{2}}(0)\right]+o\left(t^{1 / m_{n}}\right)\right\} .
\end{aligned}
$$

Here $\Gamma(\cdot)$ is the usual Gamma function; recall that $\Gamma(1 / 2)=\sqrt{\pi}$. This expansion is basic in what follows.

Proof of Theorem 9. As above, let $\Gamma \subset M$ be the set of all midpoints of minimizing geodesics from $q_{1}$ to $q_{2}$, and let $N(\Gamma)$ be a sufficiently small neighborhood of $\Gamma$ (as in [11], where we indicate the conditions it must be small enough to satisfy in a moment). Now by the assumption that every minimizing geodesic from $q_{1}$ to $q_{2}$ corresponds to a singular point of type $(1, m)$ and Lemma 27, we know that $h=h_{q_{1}, q_{2}}$ has normal form

$$
h(x)=d^{2}\left(q_{1}, q_{2}\right) / 4+x_{1}^{2}+\cdots+x_{n-1}^{2}+x_{n}^{m+1}, \quad \text { for some } m \in\{1,3,5, \ldots\},
$$

at each point of $\Gamma$. Hence the points of $\Gamma$ are isolated, and since $\Gamma$ is compact, there are only finitely many points in $\Gamma$. We enumerate them as $z_{1}, \ldots, z_{k}$.

Now for each $z_{i}$, let $N_{i}$ be a neighborhood of $z_{i}$ that is small enough for there to exist coordinates on $N_{i}$ realizing the normal form of $h\left(\right.$ at $\left.z_{i}\right)$, small enough that they are disjoint, and small enough so that [11, Theorem 27] applies to $N(\Gamma)=\cup_{i=1}^{k} N_{i}$. Then applying this theorem, we get the small-time asymptotic expansion, for some $\varepsilon>0$,

$$
p_{t}\left(q_{1}, q_{2}\right)=\sum_{i=1}^{k} \int_{N_{i}}\left(\frac{2}{t}\right)^{n} e^{-h(z) / t}\left(c_{0}\left(q_{1}, z\right) c_{0}\left(z, q_{2}\right)+O(t)\right) \mu(d z)+O\left(e^{-\frac{d^{2}\left(q_{1}, q_{2}\right)+\varepsilon}{4 t}}\right) .
$$

Here the $c_{0}\left(q_{1}, \cdot\right)$ and $c_{0}\left(\cdot, q_{2}\right)$ are the leading coefficient functions in the Ben Arous expansion; for our purposes, it is enough to note that they are smooth, positive functions on $N(\Gamma)$. Further, $\mu$ is the volume measure on $M$, and we recall that the " $O(t)$ " term in each integral is uniform over $N_{i}$. We now treat each term in this sum.

Let $m\left(z_{i}\right)$ be such that the minimizing geodesic through $z_{i}$ corresponds to a singularity of type $\left(1, m\left(z_{i}\right)\right)$; we choose this notation to avoid too much confusion with the notation used in Equation (11). (Also, it is again clear that we must have $m\left(z_{i}\right) \in\{1,3,5, \ldots\}$.) Thus we have coordinates $\left(x_{1}, \ldots, x_{n}\right)$ on $N_{i}$ such that

$$
h\left(x_{1}, \ldots, x_{n}\right)=d^{2}\left(q_{1}, q_{2}\right) / 4+x_{1}^{2}+\cdots+x_{n-1}^{2}+x_{n}^{m\left(z_{i}\right)+1} .
$$

Also, because $\mu$ is a smooth measure, we have that $\mu(d z)=F_{i}(x) d x_{1} \cdots d x_{n}$ for some smooth, positive function $F_{i}$ (on $N_{i}$ ). Writing the integral over $N_{i}$ in these coordinates and applying 
Equation (11) (here $m_{1}=\cdots=m_{n-1}=1$ and $\left.m_{n}=\left(m\left(z_{i}\right)+1\right) / 2\right)$, we have (keeping only the leading term of the expansion)

$$
\begin{aligned}
& \int_{N_{i}}\left(\frac{2}{t}\right)^{n} e^{-h(z) / t}\left(c_{0}\left(q_{1}, z\right) c_{0}\left(z, q_{2}\right)+O(t)\right) \mu(d z)=\left(\frac{2}{t}\right)^{n} \exp \left[\frac{-d^{2}\left(q_{1}, q_{2}\right)}{4 t}\right] \times \\
& t^{\frac{n-1}{2}+\frac{1}{m\left(z_{i}\right)+1}}\left\{\left[F_{i}\left(z_{i}\right)\left(c_{0}\left(q_{1}, z_{i}\right) c_{0}\left(z_{i}, q_{2}\right)+O(t)\right)\right] \Gamma\left(\frac{1}{2}\right)^{n-1} \frac{\Gamma\left(\frac{1}{m\left(z_{i}\right)+1}\right)}{\frac{m\left(z_{i}\right)+1}{2}}+O\left(t^{\frac{2}{m\left(z_{i}\right)+1}}\right)\right\} .
\end{aligned}
$$

Here we have used the uniformity of the "O(t)" to control its integral. Of course, we easily simplify the above to find

$$
\begin{aligned}
& \int_{N_{i}}\left(\frac{2}{t}\right)^{n} e^{-h(z) / t}\left(c_{0}(x, z) c_{0}(z, y)+O(t)\right) \mu(d z)= \\
& \quad \exp \left[\frac{-d^{2}\left(q_{1}, q_{2}\right)}{4 t}\right] \frac{1}{t^{\frac{n+1}{2}-\frac{1}{m\left(z_{i}\right)+1}}}\left[C_{i}+O\left(t^{\frac{2}{m\left(z_{i}\right)+1}}\right)\right] \\
& \text { where } \quad C_{i}=F_{i}\left(z_{i}\right) c_{0}\left(q_{1}, z_{i}\right) c_{0}\left(z_{i}, q_{2}\right) \frac{4}{m\left(z_{i}\right)+1}(4 \pi)^{\frac{n-1}{2}} \Gamma\left(\frac{1}{m\left(z_{i}\right)+1}\right) .
\end{aligned}
$$

Here the positivity of $C_{i}$ follows from the positivity of $F$ and the $c_{0}$ on $N_{i}$ and the positivity of the $\Gamma$-function on the positive reals.

Returning to Equation (12), we see that $p_{t}\left(q_{1}, q_{2}\right)$ has asymptotic expansion given by a sum of $k$ terms of the form given in Equation (13), for various values of $m\left(z_{i}\right)$. It is clear that the resulting sum is asymptotically dominated by those terms with the largest value of $m\left(z_{i}\right)$. Indeed, in the notation of the theorem, $\ell$ is exactly this largest value. Thus, we find that

$$
p_{t}\left(q_{1}, q_{2}\right)=\frac{C+O\left(t^{\frac{2}{\ell+1}}\right)}{t^{\frac{n+1}{2}-\frac{1}{\ell+1}}} e^{-d^{2}\left(q_{1}, q_{2}\right) / 4 t},
$$

where $C>0$ is the sum of the $C_{i}$ corresponding to geodesics (where the geodesics are indexed by the $z_{i}$ ) which give singularities of type $(1, \ell$ ) (and by assumption, there is at least one such geodesic).

Proof of Corollary [6. By Theorem 5, every minimizing geodesic from $q_{1}$ to $q_{2}$ is either non-conjugate, which corresponds to a non-singular point, $A_{3}$-conjugate, which corresponds to a singularity of type $(1,3)$ by Lemma 29, or $A_{5}$-conjugate, which corresponds to a singularity of type $(1,5)$, again by Lemma 29. Now case (i) of the corollary follows by [11, Corollary 3], case (ii) follows by applying Theorem 9 with $\ell=3$, and case (iii) follows by applying Theorem 9 with $\ell=5$.

Proof of Corollary 0 . By the results on the cut locus of a generic 3D contact sub-Riemannian structure near the diagonal (see Section 1.2), for every $q_{2}$ sufficiently close to $q_{1}$, every minimizing geodesic from $q_{1}$ to $q_{2}$ is either non-conjugate (corresponding to a non-singular point) or $A_{3^{-}}$ conjugate (corresponding to a singularity of type $(1,3)$ by Lemma 29). Thus case (i) of the corollary follows by [11, Corollary 3]. Also, case (ii) of the corollary follows by applying Theorem 9 with $n=3$ and $\ell=3$ and from the earlier observation that $A_{3}$-conjugate geodesics arise arbitrarily close to $q_{1}$. 
Proof of Corollary 8. The proof is almost identical to that of Corollary 7. In particular, we again already know that, for $q_{1}$ as in the corollary and $q_{2}$ sufficiently close to $q_{1}$, every minimizing geodesic from $q_{1}$ to $q_{2}$ is either non-singular or $A_{3}$-conjugate (and of type $(1,3)$ ), and that there are points arbitrarily close to $q_{1}$ for which the second possibility occurs. Thus case (i) of the corollary follows by applying [11, Corollary 3], and case (ii) of the corollary follows by applying Theorem 9 with $n=4$ and $\ell=3$.

\section{Proof of the complementary results}

\subsection{Proof of Theorem 11}

Lemma 30. Let $f$ be a smooth function defined on a neighborhood of the origin in $\mathbb{R}^{n}$. Suppose that $f$ has an isolated local minimum at the origin, and that the Hessian of $f$ at the origin has rank $n-r$ for some $r \in\{0,1, \ldots, n-1\}$. Then there exist a system of coordinates $x=\left(x_{1}, \ldots, x_{n}\right)$ around the origin such that, in some neighborhood of the origin,

$$
x_{1}^{2}+\cdots+x_{n-r}^{2} \leq f(x)-f(0) \leq x_{1}^{2}+\cdots+x_{n-r}^{2}+x_{n-r+1}^{4}+\cdots+x_{n}^{4} .
$$

Proof. It's clear that $f-f(0)$ satisfies the assumptions of Lemma 24, and thus there are coordinates $\left(u_{1}, \ldots, u_{n}\right)$ near the origin and a smooth function $\varphi: \mathbb{R}^{k} \rightarrow \mathbb{R}$ such that, in some neighborhood of the origin

$$
f\left(u_{1}, \ldots, u_{n}\right)-f(0)=u_{1}^{2}+\cdots+u_{n-r}^{2}+\varphi\left(u_{n-r+1}, \ldots, u_{n}\right) .
$$

Further, $\varphi$ must have an isolated local minimum of 0 in some neighborhood of the origin of $\mathbb{R}^{k}$.

Next, we wish to show that, for some $C>0, \varphi\left(u_{n-r+1}, \ldots, u_{n}\right)<C\left(u_{n-r+1}^{4}+\cdots+u_{n}^{4}\right)$ (assuming $r \geq 1$, otherwise we consider this to be vacuously true). To simplify notation, let $y_{1}=u_{n-r+1}, \ldots, y_{r}=u_{n}$. We know that $\varphi$ is smooth, has a local minimum of 0 at the origin, and its Hessian at the origin vanishes. Thus, if we write $\varphi$ as a third-order Taylor series (with remainder) around the origin, the linear and quadratic terms all vanish. If we let $y=\left(y_{1}, \ldots, y_{r}\right)$, we have $\varphi(y)=P_{3}(y)+P_{4}(y)+R_{5}(y)$ where $P_{3}$ and $P_{4}$ are homogeneous polynomials of degree 3 and 4 , respectively, and $R_{5}$ is a smooth function such that $R_{5}(y)=o\left(\|y\|^{4}\right)$.

For any monomial of degree four $y_{i} y_{j} y_{k} y_{l}$, it is clear that $y_{i} y_{j} y_{k} y_{l} \leq \sum_{i=1}^{r} y_{i}^{4}$. Hence there exists a constant $C_{4}>0$ such that $\left|P_{4}(y)\right| \leq C_{4} \sum_{i} y_{i}^{4}$. Moreover, since $\|y\|^{4}=\left(\sum_{i} y_{i}^{2}\right)^{2}$ it is clear that there exists a constant $C_{5}>0$ such that $\|y\|^{4} \leq C_{5} \sum_{i} y_{i}^{4}$ and hence $R_{5}(y) \leq C_{5} \sum_{i} y_{i}^{4}$ in a neighborhood of the origin. To complete the argument, we wish to prove that $P_{3}=0$. Assume by contradiction that there exists $y$ such that $P_{3}(y) \neq 0$. Hence for $t \in \mathbb{R}, \varphi(t y)=t^{3} P_{3}(y)+o\left(t^{3}\right)$. If $P_{3}(y)>0$ (resp. $<0$ ) then, for $t$ small enough and negative (resp. positive), $\varphi(t y)<0$ which contradicts the hypotheses.

We conclude that $\varphi(y)=P_{4}(y)+R_{5}(y)$, and the previous considerations imply that there exists $C$ such that, in a neighborhood of the origin, $0 \leq \varphi(y) \leq C \sum_{i=1}^{r} y_{i}^{4}$, as desired. Thus, near the origin,

$$
u_{1}^{2}+\cdots+u_{n-r}^{2} \leq f(u)-f(0) \leq u_{1}^{2}+\cdots+u_{n-r}^{2}+C\left(u_{n-r+1}^{4}+\cdots+u_{n}^{4}\right) .
$$

Letting each $x_{i}$ be an appropriate rescaling of $u_{i}$ then gives a system of coordinates as claimed in the lemma. 
Proof of Theorem 11. Because there is a unique minimizing geodesic from $q_{1}$ to $q_{2}$, we know that $h=h_{q_{1}, q_{2}}$ has a unique minimum at the midpoint of this geodesic. Further, from Theorem 25, we see that the Hessian of $h$ at this midpoint has rank $n-r$. If, as usual, we let $N(\Gamma)$ be a (sufficiently small) neighborhood of this midpoint, then Lemma 30 applies to $h-\frac{1}{4} d^{2}\left(q_{1}, q_{2}\right)$, and we conclude that there are coordinates on $N(\Gamma)$ such that

$$
x_{1}^{2}+\cdots+x_{n-r}^{2} \leq h(x)-\frac{1}{4} d^{2}\left(q_{1}, q_{2}\right) \leq x_{1}^{2}+\cdots+x_{n-r}^{2}+x_{n-r+1}^{4}+\cdots+x_{n}^{4} .
$$

Now we can apply Theorem 27 of [11] to get the small-time asymptotic expansion

$$
p_{t}\left(q_{1}, q_{2}\right)=\int_{N(\Gamma)}\left(\frac{2}{t}\right)^{n} e^{-h(z) / t}\left(c_{0}\left(q_{1}, z\right) c_{0}\left(z, q_{2}\right)+O(t)\right) \mu(d z) .
$$

Here the $c_{0}\left(q_{1}, \cdot\right)$ and $c_{0}\left(\cdot, q_{2}\right)$ are smooth, positive functions on $N(\Gamma), \mu$ is the volume measure on $M$, and the "O(t)" term is uniform. Estimating $h$ from both sides in the $x$-coordinates gives

$$
\begin{aligned}
p_{t}\left(q_{1}, q_{2}\right) \leq & \int_{N(\Gamma)}\left(\frac{2}{t}\right)^{n} \exp \left[-\frac{h(0)+x_{1}^{2}+\cdots+x_{n-r}^{2}}{t}\right]\left(c_{0}\left(q_{1}, x\right) c_{0}\left(x, q_{2}\right)+O(t)\right) \mu(d x) \\
\text { and } \quad p_{t}\left(q_{1}, q_{2}\right) \geq & \int_{N(\Gamma)}\left(\frac{2}{t}\right)^{n} \exp \left[-\frac{h(0)+x_{1}^{2}+\cdots+x_{n-r}^{2}+x_{n-r+1}^{4}+\cdots+x_{n}^{4}}{t}\right] \\
& \times\left(c_{0}\left(q_{1}, x\right) c_{0}\left(x, q_{2}\right)+O(t)\right) \mu(d x) .
\end{aligned}
$$

Let $F(x)$ be the density of $\mu$ with respect to $d x_{1} \cdots d x_{n}$; then $F$ is smooth and positive.

The idea is to apply Equation (11) to the right-hand side of each of the above in order to determine the leading term in the asymptotic expansions. Starting with the first equation, note that we are free to make $N(\Gamma)$ smaller without affecting the asymptotics. Thus, assume that it is of the form $x_{i} \in(-\varepsilon, \varepsilon)$ for all $i$. Then we can use Fubini's theorem to write

$$
\begin{aligned}
& \int_{N(\Gamma)}\left(\frac{2}{t}\right)^{n} \exp \left[-\frac{h(0)+x_{1}^{2}+\cdots+x_{n-r}^{2}}{t}\right]\left(c_{0}\left(q_{1}, x\right) c_{0}\left(x, q_{2}\right)+O(t)\right) \mu(d x) \\
& =\left(\frac{2}{t}\right)^{n} e^{-d^{2}\left(q_{1}, q_{2}\right) / 4 t} \int_{(-\varepsilon, \varepsilon)^{n-r}} \exp \left[-\frac{x_{1}^{2}+\cdots+x_{n-r}^{2}}{t}\right] \times \\
& \quad\left\{\int_{(-\varepsilon, \varepsilon)^{m}}\left(c_{0}\left(q_{1}, x\right) c_{0}\left(x, q_{2}\right)+O(t)\right) F(x) d x_{n-r+1} \cdots d x_{n}\right\} d x_{1} \cdots d x_{n-r}
\end{aligned}
$$

We see that the inner integral is just some smooth, positive function, call it $g\left(x_{1}, \ldots, x_{n-r}\right)$, plus a uniform $O(t)$. Then applying Equation (11) to the outer Laplace integral, we find

$$
\begin{aligned}
p_{t}\left(q_{1}, q_{2}\right) & \leq\left(\frac{2}{t}\right)^{n} e^{-d^{2}\left(q_{1}, q_{2}\right) / 4 t} t^{\frac{n-r}{2}} \Gamma\left(\frac{1}{2}\right)^{n-r}[g(0)+O(t)] \\
& =\frac{C+O(t)}{t^{\frac{n}{2}+\frac{r}{2}}} e^{-d^{2}\left(q_{1}, q_{2}\right) / 4 t}
\end{aligned}
$$

for some $C>0$. 
The second equation is a bit simpler, since we don't need to split the integral. We have

$$
\begin{aligned}
& p_{t}\left(q_{1}, q_{2}\right) \geq \int_{N(\Gamma)}\left(\frac{2}{t}\right)^{n} \exp \left[-\frac{h(0)+x_{1}^{2}+\cdots+x_{n-r}^{2}+x_{n-r+1}^{4}+\cdots+x_{n}^{4}}{t}\right] \\
& \quad \times\left(c_{0}\left(q_{1}, x\right) c_{0}\left(x, q_{2}\right)+O(t)\right) \mu(d x) \\
& =\left(\frac{2}{t}\right)^{n} e^{-d^{2}\left(q_{1}, q_{2}\right) / 4 t} t^{\frac{n-r}{2}+\frac{r}{4}}\left[\Gamma\left(\frac{1}{2}\right)^{n-r} \frac{\Gamma\left(\frac{1}{4}\right)^{r}}{2^{r}} c_{0}\left(q_{1}, 0\right) c_{0}\left(0, q_{2}\right) F(0)+O\left(t^{1 / 2}\right)\right] \\
& =\frac{\tilde{C}+O\left(t^{1 / 2}\right)}{t^{\frac{n}{2}+\frac{r}{4}}} e^{-d^{2}\left(q_{1}, q_{2}\right) / 4 t}
\end{aligned}
$$

for some $\tilde{C}>0$.

Combining these two estimates on $p_{t}\left(q_{1}, q_{2}\right)$ and using the definition of "big- $O$ " shows that we can find constant $C_{1}, C_{2}, t_{0}>0$ such that the conclusion of the theorem holds.

\subsection{Proof of Theorem 13}

The proof consists of the construction of the family of metrics the existence of which is claimed in the theorem. The essential idea is that the local structure of $h_{q_{1}, q_{2}}$ is determined by the order of contact of the spheres of radius $d\left(q_{1}, q_{2}\right) / 2$ centered at $q_{1}$ and $q_{2}$. (These spheres are tangent exactly at points in $\Gamma$.)

In $\mathbb{R}^{2}$, consider a neighborhood $U$ of the segment of the $y$-axis from $(0,-1 / 2)$ to $(0,1 / 2)$ (we will eventually take these points to be $q_{1}$ and $q_{2}$ ). We wish to put a metric on this neighborhood such that the resulting heat kernel for these two points has the desired small-time expansion. The proof is somewhat involved, so we divide it into four steps.

Step 1: In this step, our task is to indicate the local structure of the geodesics on this neighborhood.

Let $\xi$ be the piece of the circle of radius $1 / 2$ around $(0,1 / 2)$ contained in $U$ and passing through $(0,0)$. That is, $\xi$ is just the graph of

$$
y(x)=\frac{1}{2}-\sqrt{\frac{1}{4}-x^{2}}=x^{2}+x^{4}+2 x^{6}+5 x^{8}+\cdots
$$

in some neighborhood of the origin (note that every term $x^{2 k}$ appears with non-zero coefficient). Next, for any integer $\eta$, with $\eta \geq 3$, let $\gamma_{\eta}$ be the curve given by the graph, near the origin, of the first $\eta-1$ terms in this power series; for example,

$$
\gamma_{3}=\left\{(x, y): y=x^{2}+x^{4} \text { for } x \in(-\varepsilon, \varepsilon)\right\}
$$

The important point is that $\xi$ and $\gamma_{\eta}$ are tangent to order $2 \eta-1$ but not to order $2 \eta$ at the origin.

Next, we describe the desired geodesics starting from $(0,-1 / 2)$. Let $f_{\theta}(s)$, for $\theta \in[0,2 \pi)$ and $s \in[0,1]$ be a family of curves (indexed by $\theta$ ) with the following properties. We have that $f_{\theta}(0)=(0,-1 / 2)$ for all $\theta$, and in a neighborhood of $(0,-1 / 2)$, the curves (for small $s$ ) are the usual radial lines coming from $(0,-1 / 2)$, parametrized by $\theta$ as usual, except that for convenience we let $\theta=0$ correspond to the ray upward along the $y$-axis and we let $\theta$ increase in the clockwise direction. Further, we let $f_{0}(s)$ (for $s \in[0,1]$, of course) be the segment of the $y$-axis from $(0,-1 / 2)$ to $(0,0)$. To extend the rest of the curves, we first insist that $f$, as a function of $\theta$ and $s$, gives a 
smooth diffeomorphism onto the portion of $U$ below (and including) $\gamma_{\eta}$, except of course for $s=0$. For simplicity, we assume that $f$ is symmetric with respect to reflection across the $y$-axis, that is, taking $\theta$ to $-\theta$ gives a curve which is the reflection of $f_{\theta}$ across the $y$-axis. Next, for all $\theta$ near 0 , the curve $f_{\theta}$ should intersect $\gamma_{\eta}$ perpendicularly (the point of intersection occurring when $s=1$ ), and further, for $s$ near $1, f_{\theta}(s)$ should be straight (with respect to the Euclidean metric, so that near $\gamma_{\eta}, f_{\theta}$ corresponds with the normal lines to $\gamma_{\eta}$ ).

For future use, let $\sigma$ be the arclength parameter on $\gamma_{\eta}$, normalized so that $\gamma_{\eta}(0)=(0,0)$ and the $x$-coordinate increases as $\sigma$ increases. Since $f_{\theta}(1)$ for $\theta$ near 0 also provides a parametrization of $\gamma_{\eta}$, we let $\sigma(\theta)$ denote the induced change of coordinates. Our normalizations are such that $\sigma(0)=0$. Further, by the assumptions that $f$ gives a diffeomorphism and is symmetric (as discussed above), we see that $\sigma^{\prime}(0)>0$ and $\sigma^{\prime \prime}(0)=0$. Finally, note that we can prescribe any positive value for $\sigma^{\prime}(0)$ and any real value for $\sigma^{\prime \prime \prime}(0)$, in the sense that we can find a family of curves $f$ that realize these values.

Step 2: As mentioned, the curves $f_{\theta}(s)$ are intended to be the geodesic rays starting from $(0,-1 / 2)$, relative to some metric on $U$. In this step, we describe this metric.

In particular, we take a smooth metric on $U$ with the following properties. The geodesic rays from $(0,-1 / 2)$ coincide with the curves $f_{\theta}$ until these curves hit either the boundary of $U$ or $\gamma_{\eta}$. Near $\gamma_{\eta}$, these geodesic rays are straight lines (hence Euclidean geodesics as well). The metric is Euclidean above and in a neighborhood of $\gamma_{\eta}$, and thus these geodesics continue as straight lines, normal to $\gamma_{\eta}$, on the part of $U$ above $\gamma_{\eta}$. If $r$ is the distance function from $(0,-1 / 2),(r, \theta)$ for $\theta$ as above gives polar coordinates around $(0,-1 / 2)$ (with the above convention for $\theta$ ), and $\gamma_{\eta}$ coincides, near $(0,0)$, with the locus of points that are distance $1 / 2$ from $(0,-1 / 2)$. Finally, this metric on $U$ is symmetric with respect to reflection about the $y$-axis. (That such a metric exists is a straightforward exercise, and we now assume that $U$ is equipped with some such metric.)

Also, $\xi$ coincides, near $(0,0)$, with the locus of points that are distance $1 / 2$ from $(0,1 / 2)$. Since $\xi$ and $\gamma_{\eta}$ meet only at $(0,0)$, we see that the unique minimizing geodesic from $(0,-1 / 2)$ to $(0,1 / 2)$ is the intervening segment of the $y$-axis, and these points are distance 1 from each other.

Finally, we include $U$ isometrically in the smooth 2-sphere (with some smooth metric), in such a way that the only minimizing geodesic from $(0,-1 / 2)$ to $(0,1 / 2)$ remains this segment of the $y$-axis. Having done this, it is clear that the small-time asymptotics of the heat kernel relative to $(0,-1 / 2)$ and $(0,1 / 2)$ depend only on the metric in a neighborhood of this segment of the $y$-axis, so we can again restrict our attention to $U$. We will take $q_{1}$ to be $(0,-1 / 2)$ and $q_{2}$ to be $(0,1 / 2)$.

Step 3: Having constructed our metric (or more accurately, family of metrics), the next step is to understand $h=h_{q_{1}, q_{2}}$ near $(0,0)$. We know that $h$ has a unique minimum at $(0,0)$, where it equals $1 / 4$. We know that the Hessian of $h$, restricted to the direction along the $y$-axis, is non-degenerate, so Lemma 24 implies that there are coordinates $\left(u_{1}, u_{2}\right)$ around $(0,0)$ such that $h=\frac{1}{4}+u_{1}^{2}+\varphi\left(u_{2}\right)$, for some smooth function $\varphi$. Next, note that, for $(x, y)$ near $(0,0)$, the distance from $(0,1 / 2)$ to $(x, y)$ can be determined solely from the distance to $\xi$, in the Euclidean metric (and whether $(x, y)$ is above or below $\xi$ ), and similarly for $(0,-1 / 2)$ and $\gamma_{\eta}$. In particular, near $(0,0), h(x, y)$ is completely determined by $\xi$ and $\gamma_{\eta}$ (and the fact that our metric is Euclidean near $(0,0))$. Thus, $h$ depends on $\eta$, but not on $\sigma(\theta)$. Using this relatively simple structure for $h$ and the fact that $\xi$ and $\gamma_{\eta}$ are tangent to order $2 \eta-1$ but not to order $2 \eta$ at the origin, we see that there is some constant $C>1$ such that, if $(x, y)$ is near $(0,0)$ and between $\xi$ and $\gamma_{\eta}$

$$
\frac{1}{C} x^{2 \eta}<h(x, y)-\frac{1}{4}<C x^{2 \eta} .
$$


It follows that, after possibly re-parametrizing $u_{2}$, we have $h=\frac{1}{4}+u_{1}^{2}+u_{2}^{2 \eta}$.

Step 4: In this step, we determine the asymptotic expansion, and conclude the proof.

We have just seen that the unique minimizing geodesic from $q_{1}=(0,-1 / 2)$ to $q_{2}=(0,1 / 2)$ has a singularity of type $(1,2 \eta-1)$. Thus, Theorem 9 applies, but we want a more detailed description of the asymptotic expansion. We apply Equation (11), as in the proof of Theorem 9, except that now we keep track of the coefficients of the first two terms of the expansion. From this, we have

$$
\begin{aligned}
p_{t}\left(q_{1}, q_{2}\right)= & e^{-1 / 4 t} \frac{1}{t^{(3 \eta-1) / 2 \eta}}\left\{K F(0,0) c_{0}((0,-1 / 2),(0,0)) c_{0}((0,0),(0,1 / 2))\right. \\
& \left.+t^{1 / \eta} \tilde{K} \frac{\partial^{2}}{\partial u_{2}^{2}}\left[F(\cdot) c_{0}((0,-1 / 2), \cdot) c_{0}(\cdot,(0,1 / 2))\right]_{(0,0)}+o\left(t^{1 / \eta}\right)\right\},
\end{aligned}
$$

where $K$ and $\tilde{K}$ are some positive constants depending only on $\eta$. Here we recall that $F\left(u_{1}, u_{2}\right)$ is the density of Lebesgue measure $d x d y$ with respect to $d u_{1} d u_{2}$, and that the $c_{0}$ are the coefficients of the leading term in the Minakshishundaram-Pleijel expansion. All three are smooth, positive functions. As our discussion of the coordinates $\left(u_{1}, u_{2}\right)$ makes clear, $F$ depends only on $\eta$ (via $\gamma_{\eta}$ ), and not on $\sigma(\theta)$. This property is sufficient for us here, so we make no effort to compute it more explicitly. Next, $c_{0}$ is given by the reciprocal of the square-root of the Jacobian of the exponential map (see, for example, Section 5.1 of Hsu's book 22] for further discussion). Since the metric is just the Euclidean metric near the minimizing geodesic from $(0,1 / 2)$ to $(0,0)$ (recall that this geodesic is just a segment of the $y$-axis), we see that $c_{0}((x, y),(0,1 / 2))=1$ for all $(x, y)$ near $(0,0)$.

Finally, we need to understand $c_{0}((0,-1 / 2),(x, y))$ near the origin. In polar coordinates around $(0,-1 / 2)$, the (Euclidean) volume form on the tangent space is $r d r d \theta$, which is just $1 / 2 d r d \theta$ when $r=1 / 2$, while the volume form induced by the metric on $U$ (which is Euclidean for different coordinates) is, again when $r=1 / 2$, given by $\sigma^{\prime}(\theta) d r d \theta$. Thus, if $(x, y)$ is given in polar coordinates by $(1 / 2, \theta)$ for $\theta$ near 0 , we have $c_{0}((0,-1 / 2),(x, y))=\sqrt{1 / 2 \sigma^{\prime}(\theta)}$. It follows that the leading coefficient in the expansion (14) is

$$
K \cdot F(0,0) / \sqrt{2 \sigma^{\prime}(0)} .
$$

Since $K F(0,0)$ is some positive constant depending only on $\eta$ (given our general construction of the metric), we can make this be any positive real by prescribing the appropriate (positive) value for $\sigma^{\prime}(0)$, and we do this so as to get the $\alpha$ we want in the expansion. Here, of course, we recall our earlier observation that we are able to choose $\sigma^{\prime}(0)$ in precisely this way.

We now consider the second term in the expansion. It's clear from our discussion of the $\left(u_{1}, u_{2}\right)$ coordinates that the curve $u_{1}=0$ is squeezed between $\xi$ and $\gamma_{\eta}$. Hence $\partial u_{2}$ at $(0,0)$ is parallel to the $x$-axis. So by symmetry, the first derivatives of $F$ and the $c_{0}$ 's all vanish at the origin.

Then using all of this plus the fact that $c_{0}((x, y),(0,1 / 2))$ is locally constant, we see that

$$
\frac{\partial^{2}}{\partial u_{2}^{2}}\left[F(\cdot) c_{0}((0,-1 / 2), \cdot) c_{0}(\cdot,(0,1 / 2))\right]_{(0,0)}=\sqrt{\frac{1}{2 \sigma^{\prime}(0)}} \frac{\partial^{2}}{\partial u_{2}^{2}} F(0,0)+F(0,0) \frac{\partial^{2}}{\partial u_{2}^{2}} \sqrt{\frac{1}{2 \sigma^{\prime}(0)}} .
$$

Because the curve $u_{1}=0$ is squeezed between $\xi$ and $\gamma_{\eta}$ and $\xi$ and $\gamma_{\eta}$ are tangent to at least order 5 , we see that $u_{2}$ is a re-parametrization of $\gamma_{\eta}$ up to more than order 2 (at the origin), and thus it

\footnotetext{
${ }^{3}$ In fact, it's not hard to show that the curve $u_{1}=0$ is exactly the curve of equidistant points, which is squeezed between $\xi$ and $\gamma_{\eta}$, and thus give a more concrete description of the $\left(u_{1}, u_{2}\right)$ coordinates. But this isn't necessary and would only make this long construction even longer.
} 
makes sense to compute

$$
\frac{\partial^{2}}{\partial u_{2}^{2}} \sigma^{\prime}(0)=\sigma^{\prime \prime \prime}(0)\left(\frac{\partial \theta}{\partial u_{2}}(0)\right)^{2}+\sigma^{\prime \prime}(0) \frac{\partial^{2} \theta}{\partial u_{2}^{2}}(0)=\sigma^{\prime \prime \prime}(0)\left(\frac{\partial \theta}{\partial u_{2}}(0)\right)^{2},
$$

where we've used that $\sigma^{\prime \prime}(0)$ vanishes by symmetry. Since $u_{2}(\sigma)=c \sigma+O\left(\sigma^{2}\right)$ for some $c>0$ depending only on $\eta$, we are able to see that

$$
\frac{\partial^{2}}{\partial u_{2}^{2}} \sqrt{\frac{1}{2 \sigma^{\prime}(0)}}=-\frac{1}{2 \sqrt{2}} \frac{1}{\sigma^{\prime}(0)^{3 / 2}} \sigma^{\prime \prime \prime}(0)\left(\frac{1}{c \sigma^{\prime}(0)}\right)^{2} .
$$

It follows that the coefficient of the second term in the expansion (14) is

$$
\tilde{K}\left\{\sqrt{\frac{1}{2 \sigma^{\prime}(0)}} \frac{\partial^{2} F}{\partial u_{2}^{2}}(0,0)-F(0,0) \frac{1}{2 \sqrt{2}} \frac{1}{c^{2} \sigma^{\prime}(0)^{7 / 2}} \sigma^{\prime \prime \prime}(0)\right\} .
$$

Since we've already prescribed $\sigma^{\prime}(0)$, we see that there is a unique (real) value for $\sigma^{\prime \prime \prime}(0)$ that will make this expression equal to any given value. Since we are free to prescribe this value, independently of everything else in the expression, we do so in order to get the desired $\beta$ in the expansion. Thus, we have produced a metric with the desired properties, proving the theorem.

Acknowledgements. This work has been supported by the European Research Council, ERC StG 2009 "GeCoMethods", contract number 239748.

\section{References}

[1] A. Agrachev, Compactness for sub-Riemannian length-minimizers and subanalyticity, Rend. Sem. Mat. Univ. Politec. Torino, 56 (1998), pp. 1-12 (2001). Control theory and its applications (Grado, 1998).

[2] A. Agrachev, D. BARIlari, AND U. Boschin, Introduction to Riemannian and sub-Riemannian geometry (Lecture Notes), http://people.sissa.it/agrachev/agrachev_files/notes.html, (2012).

[3] - On the Hausdorff volume in sub-Riemannian geometry, Calc. Var. and PDE's, 43 (2012), pp. 355388.

[4] A. Agrachev, U. Boscain, J.-P. Gauthier, And F. Rossi, The intrinsic hypoelliptic Laplacian and its heat kernel on unimodular Lie groups, J. Funct. Anal., 256 (2009), pp. 2621-2655.

[5] A. Agrachev And J.-P. Gauthier, On the subanalyticity of Carnot-Caratheodory distances, Ann. Inst. H. Poincaré Anal. Non Linéaire, 18 (2001), pp. 359-382.

[6] A. A. Agrachev, Exponential mappings for contact sub-Riemannian structures, J. Dynam. Control Systems, 2 (1996), pp. 321-358.

[7] A. A. Agrachev, G. Charlot, J. P. A. Gauthier, And V. M. Zakalyukin, On sub-Riemannian caustics and wave fronts for contact distributions in the three-space, J. Dynam. Control Systems, 6 (2000), pp. 365-395.

[8] A. A. Agrachev and Y. L. Sachkov, Control theory from the geometric viewpoint, vol. 87 of Encyclopaedia of Mathematical Sciences, Springer-Verlag, Berlin, 2004. Control Theory and Optimization, II. 
[9] V. I. Arnol'd, S. M. GuseI n Zade, and A. N. Varchenko, Singularities of differentiable maps. Vol. II, vol. 83 of Monographs in Mathematics, Birkhäuser Boston Inc., Boston, MA, 1988.

[10] R. Azencott, Densité des diffusions en temps petit: développements asymptotiques. I, in Seminar on probability, XVIII, vol. 1059 of Lecture Notes in Math., Springer, Berlin, 1984, pp. 402-498.

[11] D. Barilari, U. Boscain, And R. W. Neel, Small-time heat kernel asymptotics at the subRiemannian cut locus, J. Differential Geom., 92 (2012), pp. 373-416.

[12] D. Barilari and J. Jendrej, Small time heat kernel asymptotics at the cut locus on surfaces of revolution, Annales de l'Institut Henri Poincaré/Analyse non linéaire, DOI:10.1016/j.anihpc.2013.03.003, (2013).

[13] G. Ben Arous, Développement asymptotique du noyau de la chaleur hypoelliptique hors du cut-locus, Ann. Sci. École Norm. Sup. (4), 21 (1988), pp. 307-331.

[14] M. Berger, A panoramic view of Riemannian geometry, Springer-Verlag, Berlin, 2003.

[15] D. Burago, Y. Burago, and S. Ivanov, A course in metric geometry, vol. 33 of Graduate Studies in Mathematics, American Mathematical Society, Providence, RI, 2001.

[16] G. Charlot, Quasi-contact $S$ - $R$ metrics: normal form in $\mathbf{R}^{2 n}$, wave front and caustic in $\mathbf{R}^{4}$, Acta Appl. Math., 74 (2002), pp. 217-263.

[17] E.-H. C. El-Alaoui, J.-P. Gauthier, And I. Kupka, Small sub-Riemannian balls on $\mathbf{R}^{3}$, J. Dynam. Control Systems, 2 (1996), pp. 359-421.

[18] R. Estrada and R. P. Kanwal, A distributional approach to asymptotics, Birkhäuser Advanced Texts: Basler Lehrbücher. [Birkhäuser Advanced Texts: Basel Textbooks], Birkhäuser Boston Inc., Boston, MA, second ed., 2002. Theory and applications.

[19] A. Grigor'Yan, Heat kernel and analysis on manifolds, vol. 47 of AMS/IP Studies in Advanced Mathematics, American Mathematical Society, Providence, RI, 2009.

[20] D. Gromoll and W. Meyer, On differentiable functions with isolated critical points, Topology, 8 (1969), pp. 361-369.

[21] L. Hörmander, Hypoelliptic second order differential equations, Acta Math., 119 (1967), pp. 147-171.

[22] E. P. Hsu, Stochastic analysis on manifolds, vol. 38 of Graduate Studies in Mathematics, American Mathematical Society, Providence, RI, 2002.

[23] S. Janeczko and T. Mostowski, Relative generic singularities of the exponential map, Compositio Math., 96 (1995), pp. 345-370.

[24] F. KLOK, Generic singularities of the exponential map on Riemannian manifolds, Geom. Dedicata, 14 (1983), pp. 317-342.

[25] R. LÉAndre, Majoration en temps petit de la densité d'une diffusion dégénérée, Probab. Theory Related Fields, 74 (1987), pp. 289-294.

[26] — Minoration en temps petit de la densité d'une diffusion dégénérée, J. Funct. Anal., 74 (1987), pp. 399-414.

[27] — Développement asymptotique de la densité d'une diffusion dégénérée, Forum Math., 4 (1992), pp. $45-75$.

[28] S. Minakshisundaram, Eigenfunctions on Riemannian manifolds, J. Indian Math. Soc. (N.S.), 17 (1953), pp. 159-165 (1954).

[29] S. Minakshisundaram And A. Pleijel, Some properties of the eigenfunctions of the Laplace-operator on Riemannian manifolds, Canadian J. Math., 1 (1949), pp. 242-256. 
[30] S. A. Molčanov, Diffusion processes, and Riemannian geometry, Uspehi Mat. Nauk, 30 (1975), pp. 359.

[31] R. Montgomery, A tour of subriemannian geometries, their geodesics and applications, vol. 91 of Mathematical Surveys and Monographs, American Mathematical Society, Providence, RI, 2002.

[32] R. NeEL, The small-time asymptotics of the heat kernel at the cut locus, Comm. Anal. Geom., 15 (2007), pp. 845-890.

[33] R. Neel And D. Stroock, Analysis of the cut locus via the heat kernel, in Surveys in differential geometry. Vol. IX, Surv. Differ. Geom., IX, Int. Press, Somerville, MA, 2004, pp. 337-349.

[34] L. S. Pontryagin, V. G. Boltyanskit, R. V. Gamkrelidze, and E. F. Mishchenko, The mathematical theory of optimal processes, Translated from the Russian by K. N. Trirogoff; edited by L. W. Neustadt, Interscience Publishers John Wiley \& Sons, Inc. New York-London, 1962.

[35] S. Rosenberg, The Laplacian on a Riemannian manifold, vol. 31 of London Mathematical Society Student Texts, Cambridge University Press, Cambridge, 1997. An introduction to analysis on manifolds.

[36] R. S. Strichartz, Sub-Riemannian geometry, J. Differential Geom., 24 (1986), pp. 221-263.

[37] C. T. C. WALL, Geometric properties of generic differentiable manifolds, in Geometry and topology (Proc. III Latin Amer. School of Math., Inst. Mat. Pura Aplicada CNPq, Rio de Janeiro, 1976), Springer, Berlin, 1977, pp. 707-774. Lecture Notes in Math., Vol. 597.

[38] A. Weinstein, The generic conjugate locus, in Global Analysis (Proc. Sympos. Pure Math., Vol. XV, Berkeley, Calif., 1968), Amer. Math. Soc., Providence, R.I., 1970, pp. 299-301.

\section{DAVIDE BARILARI}

Université Paris Diderot - Paris 7, Institut de Mathematique de Jussieu, UMR CNRS 7586 - UFR de Mathématiques. CNRS, CMAP École Polytechnique and Équipe INRIA GECO Saclay Île-de-France, Paris.

E-mail: barilari@math.jussieu.fr

\section{UGO Boscain}

CNRS, CMAP École Polytechnique and Équipe INRIA GECO Saclay Île-de-France, Paris.

E-mail: boscain@cmap.polytechnique.fn

\section{GréGoire Charlot}

Institut Fourier, UMR 5582, Université Grenoble 1 and Équipe INRIA GECO Saclay Île-de-France, Paris.

E-mail: charlot@ujf-grenoble.fr

Robert W. NEEL

Department of Mathematics, Lehigh University, Bethlehem, PA, USA

E-mail: robert.neel@lehigh.edy 